\title{
Perception and Adoption Level of Urban Horticulture Technologies, Nairobi County, Kenya
}

\author{
Ezra Oyaro*, J. B Mukundi \\ Jomo Kenyatta University of Agriculture and Technology, Department of Horticulture
}

*Corresponding Author: Ezra Oyaro, Jomo Kenyatta University of Agriculture and Technology, Department of Horticulture

\begin{abstract}
Recent years have witnessed a significant improvement in the adoption and promotion of horticultural technologies among smallholder farmers world-wide and in particular, developing countries. This study is aimed at evaluating the socio-economic factors that significantly determine farmers' decision to adoption of horticultural practices and how knowledge transfer influences urban horticulture. A sample of 580 respondents was used, 138 in Kasarani, 195 in Mathare and 247 in Kibera. Questionnaires were used to collect data from urban farmers and an interview schedule used to collect information from farmers. Social and economic characteristics, accesses to space, access to information, business management and governance data was collected. Frequencies and percentage were used to analyze the data using Statistical Package for Social Sciences (SPSS). It was found that age is significant and has a positive relationship with technology adoption, women are mainly in charge of urban farming and the farmers are spread over all education level and the source of food is the main reason for urban horticulture. The chi-square was used to differentiate different groups and conclusions. The findings show that there is a positive relationship between occupation in peri-urban and reason for adoption of urban technologies. The study also shows that there is positive relationship between age in peri-urban and technology transfer. More than $65 \%$ of the respondents were female. There is a negative relationship between education level and technology transfer. The results from this study will enable technology implementers, policy makers and local leaders to promote appropriate technologies to the residents which will lead to increased food supply, ensure food security for active and healthy life.
\end{abstract}

Keywords: Urban horticulture, food security and technology

\section{INTRODUCTION}

Urban Horticulture is the cultivation, processing, and sale of fruits, nuts, vegetables, ornamental plants, and flowers as well as many additional services (Shyr \& Reily, 2017). The products of urban horticulture include a large variety of vegetables, cereals, flowers, ornamental trees, aromatic vegetables and mushrooms.

The significance of urban horticulture as an important and growing sector of the urban space economy can be appreciated at individual household, community, and national levels. Urban horticulture is also a source of employment, income and favors both social inclusion and reduction of gender inequalities as $65 \%$ of urban farmers are women (Orsini et al. 2013). A key challenge is developing policy, strategies and technical support mechanisms for the sustainable management of urban agricultural systems, addressing production issues and marketing needs within a broader framework of environmental planning and management, water supply and utilization schemes, and food safety assurance (FAO, 2010).

Kenya is one of the countries in East Africa with high population growth rate of $2.11 \%$ (World Bank, 2013). Most of the people work within the agricultural sector and their households depend on the harvest (World Bank Group, 2008). However, food insecurity is still a major problem and malnutrition is common in urban areas (Dubbeling, de Zeeuw and van Veenhuizen 2010). Within this reality, urban agriculture/horticulture has become a key component of the survival strategies of poorer sections of the population, while also providing a significant contribution to the urban fresh food supply chain (FAO, 2010). The Government of Kenya has outlined four priority areas for the next five years. These are agricultural and food security, affordable housing, increased share of manufacturing, and universal health coverage (World Bank, 2018). 
The world urban population is expected to surpass 8.5 billion by 2030 (UN, 2015). The urban population expansion is more pronounced in developing countries as a result of emigration from rural areas, as people flock to the cities in search of food, employment and security. The trend is accelerating, and by the year 2050 , it is expected that about $66 \%$ of the world's population will be living in cities (UN, 2014). More than 60 percent of the population of Nairobi lives in the numerous slums located around the city (UNICEF, 2014). Kibera slum is one of "the biggest slum in Africa" (Desgroppes \& Taupin, 2011). Around half million people are currently living in Kibera and the population is increasing daily (Gallaher, WinklerPrins, Njenga \& Karanja, 2015). In the slum, landslides are frequent and the unemployment rate is very high. Most of the land is dedicated to housing, and agricultural land remains scarce.

Urban dwellers face relatively high living costs of housing, transportation, health care, education, inflated food prices and cash requirements when compared to their rural equivalents (Cohen \& Garrett, 2010). Such rapid urbanization and the harsh reality of urban poverty require strategies to ensure adequate food supply and distribution systems to address escalating levels of urban food insecurity. Besides the growing demand for food, malnutrition remains central issues as poverty continues to be prevalent in many cities around the world. Specifically, it is estimated that $40 \%$ of urban inhabitants are living on less than US\$1 a day, while simultaneously $70 \%$ are living on US\$2 a day (FAO, 2012). Similarly, impoverished urban households are estimated to spend 60-80 percent of incomes on food, making them more vulnerable to food price volatility (Cohen \& Garrett, 2010). Information on the sociological and economic factors and constrains affecting and limiting consumption, production and marketing of vegetables in urban areas is only sparsely or not available at all. Various technologies for vegetable production are available but have to be modified, adapted and tested under the special urban environments. A comprehensive research is therefore needed to address the issues.

\section{RESEARCH METHODOLOGY}

\subsection{Location of the Study}

The study was conducted in Nairobi County. The County covers an area of 695.1 kilometres squared (KNBS, 2009) and it borders Kiambu, Machakos and Kajiado counties. According to the Kenya National Census that was carried out 2009, the number of people living in the county is approximated to be 3,138,369 making it one of the highly populated counties in the country (KNBS, 2010). Nairobi, the capital city of Kenya, is located 140 kilometres south of the Equator and 480 kilometres from the Indian Ocean, at around latitude $1^{\circ} \mathrm{S}$ and longitude $36^{\circ} \mathrm{E}$ (Makokha \& Shisanya, 2010). It covers an area of approximately 690 square kilometres and has a diverse physical environment. The altitude of Nairobi ranges from an average of 1500 metres in the East to approximately 1900 metres (Makokha \& Shisanya, 2010).

In the eastern, north-eastern and south-eastern parts of the city centre, new residential estates have been constructed (Makokha \& Shisanya, 2010). More open and green spaces have diminished, leading to increase in the concrete jungle within the city environment. Further, immigration into the city for employment opportunities has led to growth of spontaneous squatter settlements in many places within the city (Makokha \& Shisanya, 2010).

\subsection{Research Design}

The study adopted a descriptive survey design. Survey design was suitable for this study due to the fact that it allowed to interviewing and asking people about themselves directly as well as getting the primary data.

\subsection{Sampling Procedure}

Stratified sampling was used. The 247 respondents were selected from the 9 villages (Makina, Mashimoni, Laini Saba, Kianda, Kisumu Ndogo, Soweto East, Soweto West, Gatwekera, and Silanga) in Kibera, 195 respondents were selected from the 8 villages (3A, 4A, Gitathuru, Kiamutisya, Kosovo, KwaKariuki, Mabatini, Mashimoni) in Mathare and 138 respondents were selected from the two areas of Mwiki and Kasarani. The villages/areas acted as strata where an equal number of respondents were selected from each stratum. 


\subsection{Data Collection}

The study relied on primary data of qualitative and quantitative nature. A questionnaire was used to collect social-economic data such as age, gender and occupation. The interview was the supplementary tool to collect information from urban farmers. The farmers were required to fill in the questionnaires by ticking the boxes where appropriate.

\subsection{Data Analysis Procedures}

After data collection, questions were coded and entered on spreadsheet into the computer for analysis. Frequencies, percentages, tables and means were calculated to give a simple summary of the observations. A Chi-square test for pairs of variables was used to test the significance of the relationship between the independent and dependent variables.

\section{RESULTS AND DISCUSSION}

\subsection{Socio-Demographic Characteristics of Respondents}

Table1.The distribution of the respondents based on their demographic characteristics in urban areas

\begin{tabular}{|c|c|c|c|c|c|c|}
\hline & \multicolumn{4}{|c|}{ Urban } & \multirow{2}{*}{\multicolumn{2}{|c|}{$\begin{array}{c}\text { Peri-urban } \\
\text { Kasarani }\end{array}$}} \\
\hline & \multicolumn{2}{|c|}{ Mathare } & \multicolumn{2}{|c|}{ Kibera } & & \\
\hline $\begin{array}{l}\text { Social-economic } \\
\text { characteristics }\end{array}$ & Frequency & Percentage & Frequency & Percentage & Frequency & Percentage \\
\hline \multicolumn{7}{|l|}{ Sex } \\
\hline Male & 61 & 31.3 & 74 & 30 & 34 & 24.6 \\
\hline Female & 134 & 68.7 & 173 & 70 & 104 & 75.4 \\
\hline \multicolumn{7}{|l|}{ Age } \\
\hline Young adults & 49 & 25.1 & 64 & 25.9 & 31 & 22.5 \\
\hline Middle aged & 132 & 67.7 & 160 & 64.8 & 96 & 69.5 \\
\hline Older adults & 14 & 7.2 & 23 & 9.3 & 11 & 8 \\
\hline \multicolumn{7}{|l|}{ Marital status } \\
\hline Single & 22 & 11.3 & 40 & 16.2 & 13 & 9.4 \\
\hline Married & 144 & 73.9 & 170 & 68.8 & 98 & 71 \\
\hline Divorced/separated & 10 & 5.1 & 10 & 4.1 & 4 & 2.9 \\
\hline Widowed & 19 & 9.7 & 27 & 10.9 & 23 & 19.7 \\
\hline \multicolumn{7}{|l|}{ Education Level } \\
\hline Non formal & 16 & 8.2 & 23 & 9.3 & 8 & 5.8 \\
\hline Primary & 26 & 13.3 & 48 & 19.5 & 16 & 11.6 \\
\hline Secondary & 108 & 55.4 & 129 & 52.2 & 41 & 29.7 \\
\hline Tertiary & 45 & 23.1 & 47 & 19 & 73 & 52.9 \\
\hline \multicolumn{7}{|l|}{ Occupation } \\
\hline Trading & 87 & 44.6 & 101 & 40.9 & 48 & 34.8 \\
\hline Farming & 4 & 2.1 & 3 & 1.2 & 2 & 1.5 \\
\hline Driving & 4 & 2.1 & 8 & 3.2 & 3 & 2.2 \\
\hline Civil Service & 25 & 12.8 & 30 & 12.2 & 30 & 21.7 \\
\hline Barbing & 2 & 1 & 5 & 2 & 1 & 0.7 \\
\hline Unemployed & 28 & 14.4 & 36 & 14.6 & 16 & 11.6 \\
\hline Others & 45 & 23.1 & 64 & 25.9 & 38 & 27.5 \\
\hline \multicolumn{7}{|l|}{ Level of income } \\
\hline Less than 5,000 & 11 & 5.6 & 20 & 8.1 & 3 & 2.2 \\
\hline $5,001-10,000$ & 23 & 11.8 & 16 & 6.5 & 5 & 3.6 \\
\hline $10,001-15,000$ & 41 & 21 & 48 & 19.4 & 18 & 13 \\
\hline $15,001-20,000$ & 39 & 20 & 42 & 17 & 31 & 22.5 \\
\hline $20,001-25,000$ & 19 & 9.7 & 38 & 15.4 & 28 & 20.3 \\
\hline $25,001-30,000$ & 12 & 6.2 & 19 & 7.7 & 19 & 13.8 \\
\hline $30,001-35,000$ & 16 & 8.2 & 14 & 5.7 & 13 & 9.4 \\
\hline $35,001-40,000$ & 14 & 7.2 & 17 & 6.9 & 9 & 6.5 \\
\hline $40,001-45,000$ & 6 & 3.1 & 9 & 3.6 & 5 & 3.6 \\
\hline $45,001-50,000$ & 8 & 4.1 & 13 & 5.3 & 4 & 2.9 \\
\hline More than 50,000 & 6 & 3.1 & 11 & 4.5 & 3 & 2.2 \\
\hline
\end{tabular}

Source: Survey, August 2017 
As shown above (Table 1), it is apparent that the majority of respondents were females, for instance, in Kasarani $75.4 \%$ were females and the remainder were males (24.6\%). In Mathare, $68.7 \%$ were females and the males were $31.3 \%$, and in Kibera, $70 \%$ were females and males were $30 \%$. The marital status of respondents as shown on the table, varied from single (9.4\%), married $(71 \%)$, separated/divorced $(2.9 \%)$ to widowed $(16.7 \%)$ in Kasarani. In Mathare, $11.3 \%$ were single, married $73.9 \%$, separated/divorced $5.1 \%$ to widowed $9.7 \%$ and in Kibera, $16.2 \%$ were single, married were $68.8 \%$, separated/divorced $4.1 \%$ and widowed $10.9 \%$. It is indicated that most respondents were married; Kasarani (71\%), Mathare (73.9\%) and Kibera (68.8\%).

It is clear that the majority of the respondents are middle aged adults with $67.7 \%, 64.8 \%$ and $69.5 \%$ for Mathare, Kibera and Kasarani respectively. On the level of education, the majority (52.9\%) of the respondents had post-secondary education in the form of certificates, diplomas and degrees, followed by $29.7 \%$ with secondary education, $11.6 \%$ with primary education and $5.8 \%$ had no-formal education in Kasarani area. In Mathare, the majority (55.4\%) of the respondents had secondary education, followed by $23.1 \%$ with post-secondary education in the form of certificates, diplomas and degrees, $13.3 \%$ with primary education and only $8.2 \%$ had no-formal education. In Kibera, the majority $(52.2 \%)$ of the respondents had secondary education, followed by $19.5 \%$ with primary education, $19.0 \%$ with post-secondary education in theform of certificates, diplomas and degrees and only $8.2 \%$ had no-formal education. The average monthly income of the respondents was between Kshs. $(10,000-35,000)$ for most respondents.

\subsection{Frequencies of Respondents by Space Identified for Production of Vegetables}

Table2 (i). Frequency for space identified for growing vegetables according to social-demographic groups of respondents in Kasarani

\begin{tabular}{|c|c|c|c|c|c|c|c|c|c|c|c|}
\hline & $\begin{array}{l}\text { Socio-economic } \\
\text { characteristics }\end{array}$ & $\begin{array}{c}\text { Roofto } \\
\text { ps }\end{array}$ & $\begin{array}{c}\text { Balcone } \\
s\end{array}$ & $\begin{array}{c}\text { Vacant } \\
\text { places }\end{array}$ & \begin{tabular}{|c|} 
In \\
container \\
$\boldsymbol{s}$
\end{tabular} & $\begin{array}{c}\text { Along } \\
\text { railways }\end{array}$ & $\begin{array}{c}\text { Below } \\
\text { power } \\
\text { lines }\end{array}$ & $\begin{array}{l}\text { River } \\
\text { banks }\end{array}$ & $\begin{array}{c}\text { School } \\
\text { garden } \\
\text { S }\end{array}$ & $\begin{array}{l}\text { Road } \\
\text { strips }\end{array}$ & others \\
\hline \multirow{4}{*}{ らँ } & Males & (1) $3 \%$ & (2) $6 \%$ & (6) $18 \%$ & (5) $15 \%$ & (2) $6 \%$ & (3) $9 \%$ & (9) $27 \%$ & (0) $0 \%$ & (6) $18 \%$ & (2) $3 \%$ \\
\hline & Female $(n=104)$ & (4) $4 \%$ & (4) $4 \%$ & $(27) 26 \%$ & $(9) 9 \%$ & $(14) 14 \%$ & $(4) 4 \%$ & $(12) 12 \%$ & (3)3\% & $(18) 17 \%$ & (9) $8 \%$ \\
\hline & Total $(\mathrm{n}=138)$ & $4 \%$ & $(6) 4 \%$ & (33) $24 \%$ & $(14) 10 \%$ & $(16) 12 \%$ & $(7) 5 \%$ & $(21) 15 \%$ & $(3) 2 \%$ & $24) 17 \%$ & $(11) 8 \%$ \\
\hline & $\mathrm{P}$ value & \multicolumn{10}{|c|}{0.494} \\
\hline \multirow{4}{*}{$\underset{\infty}{\infty}$} & Young adults $(\mathrm{n}=31)$ & (1)3\% & $(1) 3 \%$ & $(7) 23 \%$ & $(5) 16 \%$ & $(3) 10 \%$ & (3)10\% & (6)19\% & (0)0\% & (5)16\% & $(0) 0 \%$ \\
\hline & & (3) $3 \%$ & $(5) 5 \%$ & (21) $22 \%$ & (9)9\% & (13) $14 \%$ & (4) $4 \%$ & (13) $14 \%$ & (3)3\% & (17) $18 \%$ & $(8) 8 \%$ \\
\hline & Older adults $(\mathrm{n}=11)$ & 1) $9 \%$ & $(0) 0 \%$ & $(5) 46 \%$ & $(0) 0 \%$ & $(0) 0 \%)$ & $(0) 0 \%$ & $18 \%$ & $(0) 0 \%$ & $18 \%$ & (1)9\% \\
\hline & $\mathrm{P}_{\mathrm{va}}$ & \multicolumn{10}{|c|}{0.465} \\
\hline \multirow{5}{*}{ 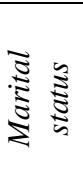 } & Single $(n$ & $(0) 0 \%$ & (1) $8 \%$ & & $(0) 0 \%$ & (1)8\% & $(0) 0 \%$ & (1) $8 \%$ & (1)8\% & (4)31\% & (2) $15 \%$ \\
\hline & Married & $3) 3 \%$ & (4) $4 \%$ & (24) $25 \%$ & (13)13\% & (12) $12 \%$ & (6) $6 \%$ & $16 \%$ & (2)2\% & $12 \%$ & (6) $6 \%$ \\
\hline & Divorced/sep & (1)25\% & $(0) 0 \%$ & & $(0) 0 \%$ & $5 \%$ & $(0) 0 \%$ & $5 \%$ & $(0) 0 \%$ & & $0) 0 \%$ \\
\hline & Widowe & (1)4\% & (1)4\% & $(5) 22 \%$ & (1) $4 \%$ & $(2) 9 \%$ & $(1) 4 \%$ & $3 \%$ & $(0) 0 \%$ & $5 \%$ & (1)4\% \\
\hline & & \multicolumn{10}{|c|}{0.598} \\
\hline \multirow{5}{*}{ 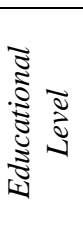 } & $\begin{array}{r}\text { Non fo } \\
\text { educatio }\end{array}$ & $(0) 0 \%$ & (1) $13 \%$ & (3) $38 \%$ & (1) $13 \%$ & (2) $25 \%$ & $(0) 0 \%$ & (1) $13 \%$ & $(0) 0 \%$ & $(0) 0 \%$ & $(0) 0 \%$ \\
\hline & Primary $(n=16)$ & (1)6\% & & & $(0) 0 \%$ & & (1)6\% & & (2)13\% & $13 \%$ & (2)13\% \\
\hline & Secon & $25 \%$ & (3)7\% & (6) & (7) $17 \%$ & $(7) 17 \%$ & (1) $2 \%$ & $0 \%$ & $(0) 0 \%$ & $2 \%$ & $(2) 5 \%$ \\
\hline & Tertiar & $(2) 3 \%$ & (2) $3 \%$ & (18) $25 \%$ & (6) $8 \%$ & $(6) 8 \%$ & $(5) 7 \%$ & (11)15\% & (1) $1 \%$ & (17)23\% & $(5) 7 \%$ \\
\hline & & \multicolumn{10}{|c|}{0.232} \\
\hline \multirow{8}{*}{ 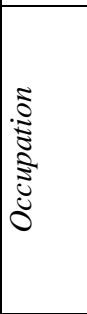 } & Trading & $(4) 8 \%$ & (1) $2 \%$ & (9)19\% & (4) $8 \%$ & $(6) 13 \%$ & (3)6\% & (7) $15 \%$ & (1) $2 \%$ & $21 \%$ & (3) $6 \%$ \\
\hline & & $(0) 0 \%$ & $(0) 0 \%$ & & (1)50\% & & (1)50\% & & $(0) 0 \%$ & $0 \%$ & $(0) 0 \%$ \\
\hline & Dri & $0) 0 \%$ & (1) $33 \%$ & & $(0) 0 \%$ & $3 \%$ & $(0) 0 \%$ & & (0)0\% & $0 \%$ & $(0) 0 \%$ \\
\hline & Civil Servi & 1)3\% & $(2) 7 \%$ & $0 \%$ & (3) $10 \%$ & $0 \%$ & (1)3\% & $27 \%$ & $(0) 0 \%$ & $10 \%$ & (3) $10 \%$ \\
\hline & Barl & $(0) 0 \%$ & $(0) 0 \%$ & $(0) 0 \%$ & $(0) 0 \%$ & $(0) 0 \%$ & $(0) 0 \%$ & $(0) 0 \%$ & $(0) 0 \%$ & (1)100\% & $(0) 0 \%$ \\
\hline & Unemploy & $(0) 0 \%$ & (2)13\% & (3) $19 \%$ & (2)13\% & (1) $6 \%$ & (1) $6 \%$ & & $(0) 0 \%$ & $(5) 31 \%$ & (1) $6 \%$ \\
\hline & Others & $0 \%$ & $(0) 0 \%$ & (14)37\% & (4) $11 \%$ & $(5) 13 \%$ & $(1) 3 \%$ & (5)13\% & $(2) 5 \%$ & $(5) 13 \%$ & $(2) 5 \%$ \\
\hline & & \multicolumn{10}{|c|}{0.243} \\
\hline \multirow{7}{*}{ 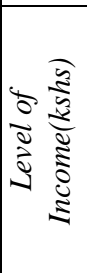 } & Less than $5,000(n=3)$ & (1)33\% & $(0) 0 \%$ & $(0) 0 \%$ & $(0) 0 \%$ & $(0) 0 \%$ & $(0) 0 \%$ & (1)33\% & $(0) 0 \%$ & (1)33\% & $(0) 0 \%$ \\
\hline & $5,001-10,000(\mathrm{n}=5)$ & $(0) 0 \%$ & $(0) 0 \%$ & (1)20\% & $(0) 0 \%$ & $(0) 0 \%$ & $(0) 0 \%$ & (1) $20 \%$ & (1)20\% & (2)40\% & $(0) 0 \%$ \\
\hline & $10,001-15,000(n=18)$ & $(0) 0 \%$ & & & (2)11\% & & (1) $6 \%$ & & $(0) 0 \%$ & (4) $22 \%$ & (2)11\% \\
\hline & $15,001-20,000(n=31)$ & (1)3\% & $(2) 7 \%$ & (7) $23 \%$ & $(2) 7 \%$ & (3) $10 \%$ & (1)3\% & (4)13\% & $(0) 0 \%$ & (7)23\% & (4) $13 \%$ \\
\hline & $20,001-25,000(n=28)$ & $(0) 0 \%$ & (1) $4 \%$ & $(8) 29 \%$ & $(2) 7 \%$ & (4)14\% & (2)7\% & (6) $21 \%$ & $(0) 0 \%$ & $(4) 14 \%$ & (1) $4 \%$ \\
\hline & $25,001-30,000(n=19)$ & (1)5\% & (3) $16 \%$ & (4) $21 \%$ & (4) $21 \%$ & (4) $21 \%$ & (1)5\% & $(1) 5 \%$ & $(0) 0 \%$ & $(1) 5 \%$ & $(0) 0 \%$ \\
\hline & $30,001-35,000(n=13)$ & (1) $8 \%$ & $(0) 0 \%$ & (3) $23 \%$ & (3) $23 \%$ & (1) $8 \%$ & $(0) 0 \%$ & (0)0\% & (1) $8 \%$ & (4)31\% & $(0) 0 \%$ \\
\hline
\end{tabular}




\begin{tabular}{|c|c|c|c|c|c|c|c|c|c|c|}
\hline $35,001-40,000(n=9)$ & $(0) 0 \%$ & $(0) 0 \%$ & (4) $44 \%$ & (1) $11 \%$ & (2) $22 \%$ & $(0) 0 \%$ & (1)11\% & $(0) 0 \%$ & (1)11\% & $(0) 0 \%$ \\
\hline $40,001-45,000(n=5)$ & (1)20\% & $(0) 0 \%$ & (1) $20 \%$ & $(0) 0 \%$ & $(0) 0 \%$ & $(0) 0 \%$ & $(1) 20 \%$ & $(0) 0 \%$ & $(0) 0 \%$ & (2) $40 \%$ \\
\hline $45,001-50,000(n=4)$ & $(0) 0 \%$ & $(0) 0 \%$ & $(0) 0 \%$ & $(0) 0 \%$ & (2)50\% & $(0) 0 \%$ & (1) $25 \%$ & (1)25\% & $(0) 0 \%$ & $(0) 0 \%$ \\
\hline More than $50,000(n=3)$ & $(0) 0 \%$ & $(0) 0 \%$ & $(0) 0 \%$ & $(0) 0 \%$ & $(0) 0 \%$ & (2)67\% & (1) $33 \%$ & $(0) 0 \%$ & $(0) 0 \%$ & $(0) 0 \%$ \\
\hline $\mathrm{P}$ value & & & & & & & & & & \\
\hline
\end{tabular}

$* \mathrm{p}<0.05$

\section{Source: survey, August 2017}

The respondents were asked about the space where they grow crops. The farmers' responses were varied. The spaces are categorized into; rooftops, balconies, vacant places, in containers, along the railways, below power lines, river banks, school gardens, road strips and others to give us a better understanding on the choice of spaces for farming in Kasarani. About $24 \%$ of the respondents did farming along the vacant places, $17 \%$ on the road strips and $15 \%$ along the water lines such as river banks and sewage lines. There was considerable variation in the choice of space for farming between farmers from different age groups (Table 2(i)). Forty six percent of older adults ( $>55$ years), did farming on vacant spaces compared to $23 \%$ of young adults $(<35$ years $)$. The choice of space also varied significantly amongst different gender groups $(p=0.494)$, age groups $(p=0.465)$, marital status $(p=0.598)$, education level $(p=0.232)$, occupation $(n=0.243)$ and level of income groups $(p=$ $0.140)$.

Table2(ii). Frequency of space identified for growing vegetables according to social-demographic groups in Mathare

\begin{tabular}{|c|c|c|c|c|c|c|c|c|c|c|c|}
\hline & $\begin{array}{l}\text { Socio-economic } \\
\text { characteristics }\end{array}$ & Rooftops & \begin{tabular}{|c|} 
Balcone \\
$\mathrm{s}$
\end{tabular} & $\begin{array}{l}\text { Vacant } \\
\text { places }\end{array}$ & $\begin{array}{c}\text { In } \\
\text { container } \\
\text { s }\end{array}$ & \begin{tabular}{|c|} 
Along \\
railway \\
$\mathrm{S}$
\end{tabular} & \begin{tabular}{|c|} 
Below \\
Power \\
lines
\end{tabular} & $\begin{array}{l}\text { River } \\
\text { banks }\end{array}$ & $\begin{array}{c}\text { School } \\
\text { garden } \\
\mathrm{S}\end{array}$ & $\begin{array}{l}\text { Road } \\
\text { strips }\end{array}$ & others \\
\hline \multirow[b]{4}{*}{$\omega^{2}$} & $\operatorname{Male}(\mathrm{n}=61)$ & $2(3 \%)$ & $4(7 \%)$ & $11(18 \%)$ & $11(18 \%)$ & $0(0 \%)$ & $2(3 \%)$ & $13(21 \%)$ & $0(0 \%)$ & $12(20 \%)$ & $6(10 \%)$ \\
\hline & Female $(n=134$ & $2(2 \%)$ & $7(5 \%)$ & $25(19 \%)$ & $21(16 \%)$ & $0(0 \%)$ & $2(2 \%)$ & $32(24 \%)$ & $2(2 \%)$ & $31(23 \%)$ & $12(9 \%)$ \\
\hline & Total $(n=195)$ & $4(2 \%)$ & $11(6 \%)$ & $36(19 \%)$ & $32(16 \%)$ & $0(0 \%)$ & $4(2 \%)$ & $45(23 \%)$ & $2(1 \%)$ & $43(22 \%)$ & $18(9 \%)$ \\
\hline & $\mathrm{P}$ value & \multicolumn{10}{|c|}{0.942} \\
\hline \multirow{4}{*}{$\underset{\infty}{\infty}$} & Young adults $(\mathrm{n}=49)$ & $1(2 \%)$ & $3(6 \%)$ & $6(12 \%)$ & $17(35 \%)$ & $0(0 \%)$ & $0(0 \%)$ & $8(16 \%)$ & $0(0 \%)$ & $9(18 \%)$ & $5(10 \%)$ \\
\hline & $\begin{array}{c}\text { Middle aged } \\
\text { adults }(\mathrm{n}=132)\end{array}$ & $3(2 \%)$ & $7(5 \%)$ & $29(22 \%)$ & $14(11 \%)$ & $0(0 \%)$ & $3(2 \%)$ & $33(25 \%)$ & $2(2 \%)$ & $31(24 \%)$ & $10(8 \%)$ \\
\hline & Older adults $(\mathrm{n}=14)$ & $0(0 \%)$ & $1(7 \%)$ & $1(7 \%)$ & $1(7 \%)$ & $0(0 \%)$ & $1(7 \%)$ & $4(29 \%)$ & $0(0 \%)$ & $3(21 \%)$ & $3(21 \%)$ \\
\hline & $\mathrm{P}$ va & \multicolumn{10}{|c|}{0.507} \\
\hline \multirow{5}{*}{ 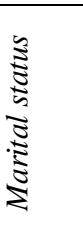 } & Single $(\mathrm{n}=22)$ & $0(0 \%)$ & $1(5 \%)$ & $7(32 \%)$ & $2(9 \%)$ & $0(0 \%)$ & $0(0 \%)$ & $2(9 \%)$ & $0(0 \%)$ & $7(32 \%)$ & $3(14 \%)$ \\
\hline & Married(n & $4(3 \%)$ & $9(6 \%)$ & $23(16 \%)$ & $23(16 \%)$ & $0(0 \%)$ & $4(3 \%)$ & $35(24 \%)$ & $2(1 \%)$ & $31(22 \%)$ & $13(9 \%)$ \\
\hline & $\begin{array}{c}\text { Divorced/separated( } \mathrm{n}= \\
10)\end{array}$ & $0(0 \%)$ & $0(0 \%)$ & $3(30 \%)$ & $3(30 \%)$ & $0(0 \%)$ & $0(0 \%)$ & $1(10 \%)$ & $0(0 \%)$ & $2(20 \%)$ & $1(10 \%)$ \\
\hline & Windowed(n=19) & $0(0 \%)$ & $1(5 \%)$ & $3(16 \%)$ & $4(21 \%)$ & $0(0 \%)$ & $0(0 \%)$ & $7(37 \%)$ & $0(0 \%)$ & $3(16 \%)$ & $1(5 \%)$ \\
\hline & & \multicolumn{10}{|c|}{0.883} \\
\hline \multirow{5}{*}{ 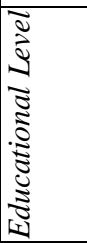 } & $\begin{array}{c}\text { Non formal } \\
\text { education }(n=16)\end{array}$ & $0(0 \%)$ & $1(6 \%)$ & $4(25 \%)$ & $2(13 \%)$ & $0(0 \%)$ & $0(0 \%)$ & $5(31 \%)$ & $0(0 \%)$ & $3(19 \%)$ & $1(6 \%)$ \\
\hline & Primary & $(4 \%)$ & $2(8 \%)$ & $4(15 \%)$ & $3(12 \%)$ & $0(0 \%)$ & $0(0 \%)$ & $1(4 \%)$ & $0(0 \%)$ & $9(35 \%)$ & $6(23 \%)$ \\
\hline & Secondary( & $1(1 \%)$ & $7(7 \%)$ & $21(19 \%)$ & $16(15 \%)$ & $0(0 \%)$ & $2(2 \%)$ & $31(29 \%)$ & $2(2 \%)$ & $20(19 \%)$ & $8(7 \%)$ \\
\hline & Tertiary $(1$ & $2(4 \%)$ & $1(2 \%)$ & $7(16 \%)$ & $11(24 \%)$ & $0(0 \%)$ & $2(4 \%)$ & $8(18 \%)$ & $0(0 \%)$ & $11(24 \%)$ & $3(7 \%)$ \\
\hline & & \multicolumn{10}{|c|}{0.328} \\
\hline \multirow{8}{*}{ 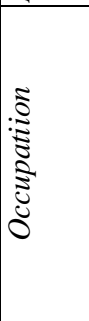 } & Tradin & $3(3 \%)$ & $4(5 \%)$ & $15(17 \%)$ & $14(16 \%)$ & $0(0 \%)$ & $0(0 \%)$ & $22(25 \%)$ & $1(1 \%)$ & $16(18 \%)$ & $12(14 \%)$ \\
\hline & Farm & $0(0 \%)$ & $2(50 \%)$ & $1(25 \%)$ & $0(0 \%)$ & $0(0 \%)$ & $0(0 \%)$ & $1(25 \%)$ & $0(0 \%)$ & $0(0 \%)$ & $0(0 \%)$ \\
\hline & $\operatorname{Driving}(\mathrm{n}=4)$ & $0(0 \%)$ & $0(0 \%)$ & $1(25 \%)$ & $3(75 \%)$ & $0(0 \%)$ & $0(0 \%)$ & $0(0 \%)$ & $0(0 \%)$ & $0(0 \%)$ & $0(0 \%)$ \\
\hline & Civil Ser & $0(0 \%)$ & $1(4 \%)$ & $7(28 \%)$ & $2(8 \%)$ & $0(0 \%)$ & $3(12 \%)$ & $4(16 \%)$ & $1(4 \%)$ & $7(28 \%)$ & $0(0 \%)$ \\
\hline & Barbing $(\mathrm{n}=2)$ & $0(0 \%)$ & $0(0 \%)$ & $0(0 \%)$ & $0(0 \%)$ & $0(0 \%)$ & $0(0 \%)$ & $0(0 \%)$ & $0(0 \%)$ & $2(100 \%)$ & $0(0 \%)$ \\
\hline & Unemployed $(\mathrm{n}=28)$ & $0(0 \%)$ & $2(7 \%)$ & $3(11 \%)$ & $5(18 \%)$ & $0(0 \%)$ & $0(0 \%)$ & $7(25 \%)$ & $0(0 \%)$ & $7(25 \%)$ & $4(14 \%)$ \\
\hline & Others $(n=45)$ & $1(2 \%)$ & $2(4 \%)$ & $9(20 \%)$ & $8(18 \%)$ & $0(0 \%)$ & $1(2 \%)$ & $11(24 \%)$ & $0(0 \%)$ & $11(24 \%)$ & $2(4 \%)$ \\
\hline & \begin{tabular}{|c|} 
P value \\
\end{tabular} & \multicolumn{10}{|c|}{0.203} \\
\hline \multirow{7}{*}{ 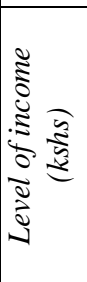 } & Less than $5,000(n=11)$ & $1(9 \%)$ & $0(0 \%)$ & $4(36 \%)$ & $2(18 \%)$ & $0(0 \%)$ & $0(0 \%)$ & $2(18 \%)$ & $0(0 \%)$ & $2(18 \%)$ & $0(0 \%)$ \\
\hline & $5,001-10,000(n=23)$ & $0(0 \%)$ & $1(4 \%)$ & $5(22 \%)$ & $1(4 \%)$ & $0(0 \%)$ & $0(0 \%)$ & $9(39 \%)$ & $0(0 \%)$ & $3(13 \%)$ & $4(17 \%)$ \\
\hline & $10,001-15,000(n=41)$ & $0(0 \%)$ & $0(0 \%)$ & $6(15 \%)$ & $6(15 \%)$ & $0(0 \%)$ & $1(2 \%)$ & $12(29 \%)$ & $1(2 \%)$ & $11(27 \%)$ & $4(10 \%)$ \\
\hline & $15,001-20,000(n=39)$ & $1(3 \%)$ & $6(15 \%)$ & $4(10 \%)$ & $3(8 \% 0$ & $0(0 \%)$ & $1(3 \%)$ & $8(21 \%)$ & $1(3 \%)$ & $10(26 \%)$ & $5(13 \%)$ \\
\hline & $20,001-25,000(n=19)$ & $2(11 \%)$ & $1(5 \%)$ & $4(21 \%)$ & $8(42 \%)$ & $0(0 \%)$ & $0(0 \%)$ & $2(11 \%)$ & $0(0 \%)$ & $1(5 \%)$ & $1(5 \%)$ \\
\hline & $25,001-30,000(n=12)$ & $0(0 \%)$ & $1(8 \%)$ & $2(17 \%)$ & $1(8 \%)$ & $0(0 \%)$ & $0(0 \%)$ & $3(25 \%)$ & $0(0 \%)$ & $5(42 \%)$ & $0(0 \%)$ \\
\hline & $30,001-35,000(n=16)$ & $0(0 \%)$ & $1(6 \%)$ & $5(31 \%)$ & $2(13 \%)$ & $0(0 \%)$ & $1(6 \%)$ & $3(19 \%)$ & $0(0 \%)$ & $3(19 \%)$ & $1(6 \%)$ \\
\hline
\end{tabular}




\begin{tabular}{|c|c|c|c|c|c|c|c|c|c|c|}
\hline $35,001-40,000(n=14)$ & $0(0 \%)$ & $0(0 \%)$ & $5(36 \%)$ & $5(36 \%)$ & $0(0 \%)$ & $0(0 \%)$ & $4(29 \%)$ & $0(0 \%)$ & $0(0 \%)$ & $0(0 \%)$ \\
\hline $40,001-45,000(n=6)$ & $0(0 \%)$ & $1(17 \%)$ & $1(17 \%)$ & $0(0 \%)$ & $0(0 \%)$ & $0(0 \%)$ & $0(0 \%)$ & $0(0 \%)$ & $3(50 \%)$ & $1(17 \%)$ \\
\hline $45,001-50,000(n=8)$ & $0(0 \%)$ & $0(0 \%)$ & $0(0 \%)$ & $4(50 \%)$ & $0(0 \%)$ & $0(0 \%)$ & $1(13 \%)$ & $0(0 \%)$ & $2(25 \%)$ & $1(13 \%)$ \\
\hline $\begin{array}{c}\text { More than } \\
50,000(n=6)\end{array}$ & $0(0 \%)$ & $0(0 \%)$ & $0(0 \%)$ & $0(0 \%)$ & $0(0 \%)$ & $1(17 \%)$ & $1(17 \%)$ & $0(0 \%)$ & $3(50 \%)$ & $1(17 \%)$ \\
\hline$P$ value & \multicolumn{10}{|c|}{0.089} \\
\hline
\end{tabular}

*p $<0.05$

\section{Source: survey, August 2017}

The respondents were asked about the space where they grow crops. The farmers' responses were varied. The spaces are categorized into; rooftops, balconies, vacant places, in containers, along the railways, below power lines, river banks, school gardens, road strips and others to give us a better understanding on the choice of spaces for farming in Mathare. About 23\% of the respondents planted along the water lines such as river banks and sewage lines, $19 \%$ did farming along the vacant places and $22 \%$ on the road strips. There was considerable variation in the choice of space for farming between farmers from different age groups (Table 2(ii) ). Seven percent $(7 \%)$ of the older adults $(>55$ years), did farming in containers compared to $35 \%$ of young adults ( $<35$ years). The choice of space also varied significantly amongst different gender groups $(\mathrm{p}=0.942)$, age groups $(\mathrm{p}=0.507)$, marital status $(\mathrm{p}=0.883)$, education level $(\mathrm{p}=0.328)$ occupation $(\mathrm{n}=0.203)$ and level of income groups $(\mathrm{p}=$ $0.089)$.

Table2(iii). Frequency of space identified for growing vegetables according to social-demographic groups in Kibera

\begin{tabular}{|c|c|c|c|c|c|c|c|c|c|c|c|}
\hline & $\begin{array}{l}\text { Socio-economic } \\
\text { characteristics }\end{array}$ & $\begin{array}{c}\text { Rooftop } \\
\text { s }\end{array}$ & Balcones & $\begin{array}{r}\text { Vacant } \\
\text { places }\end{array}$ & $\underset{\text { In }}{\text { containers }}$ & $\begin{array}{c}\text { Along } \\
\text { railways }\end{array}$ & $\begin{array}{l}\text { Below } \\
\text { power } \\
\text { lines }\end{array}$ & $\begin{array}{l}\text { River } \\
\text { banks }\end{array}$ & \begin{tabular}{|l|} 
School \\
gardens
\end{tabular} & $\begin{array}{l}\text { Road } \\
\text { strips }\end{array}$ & others \\
\hline \multirow{4}{*}{ ڤँّ } & Male $(n=74)$ & $5(7 \%)$ & $1(1 \%)$ & $11(15 \%)$ & $11(15 \%)$ & $6(8 \%)$ & $1(1 \%)$ & $18(24 \%)$ & $1(1 \%)$ & $15(20 \%)$ & $5(7 \%)$ \\
\hline & Female $(n=173)$ & $6(4 \%)$ & $1(1 \%)$ & $37(21 \%)$ & $24(14 \%)$ & $19(11 \%)$ & $1(1 \%)$ & $41(24 \%)$ & $1(1 \%)$ & $32(19 \%)$ & $11(6 \%)$ \\
\hline & Total $(n=247)$ & $11(5 \%)$ & $2(1 \%)$ & $48(19 \%)$ & $35(14 \%)$ & $25(10 \%)$ & $2(1 \%)$ & $59(24 \%)$ & $2(1 \%)$ & $47(19 \%)$ & $16(7 \%)$ \\
\hline & $\mathrm{P}$ value & \multicolumn{10}{|c|}{0.904} \\
\hline \multirow{4}{*}{$\stackrel{\infty}{\infty}$} & Young adults $(\mathrm{n}=64)$ & $5(8 \%)$ & $1(2 \%)$ & $10(16 \%)$ & $9(14 \%)$ & $10(16 \%)$ & $0(0 \%)$ & $13(20 \%)$ & $1(2 \%)$ & $11(17 \%)$ & $4(6 \%)$ \\
\hline & Middle aged $(n=160)$ & $5(3 \%)$ & $1(1 \%)$ & $31(19 \%)$ & $22(14 \%)$ & $12(8 \%)$ & $2(1 \%)$ & $41(26 \%)$ & $1(1 \%)$ & $13(8 \%)$ & $12(8 \%)$ \\
\hline & Older adults $(\mathrm{n}=23)$ & $1(4 \%)$ & $0(0 \%)$ & $7(30 \%)$ & $4(17 \%)$ & $3(13 \%)$ & $0(0 \%)$ & $5(22 \%)$ & $0(0 \%)$ & $3(13 \%)$ & $0(0 \%)$ \\
\hline & $\mathrm{P}$ value & \multicolumn{10}{|c|}{0.688} \\
\hline \multirow{5}{*}{ 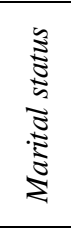 } & Single $(n=40)$ & $2(5 \%)$ & $0(0 \%)$ & $5(13 \%)$ & $3(8 \%)$ & $5(13 \%)$ & $1(3 \%)$ & $11(28 \%)$ & $0(0 \%)$ & $12(30 \%)$ & $1(3 \%)$ \\
\hline & Married(n=170) & $9(5 \%)$ & $1(1 \%)$ & $38(22 \%)$ & $25(15 \%)$ & $19(11 \%)$ & $1(1 \%)$ & $33(19 \%)$ & $1(1 \%)$ & $31(18 \%)$ & $12(7 \%)$ \\
\hline & $\begin{array}{c}\text { Divorced/separated }(\mathrm{n}=1 \\
0)\end{array}$ & $0(0 \%)$ & $0(0 \%)$ & $1(10 \%)$ & $2(20 \%)$ & $0(0 \%)$ & $0(0 \%)$ & $4(40 \%)$ & $0(0 \%)$ & $2(20 \%)$ & $1(10 \%)$ \\
\hline & Widowed $(n=27)$ & $0(0 \%)$ & $1(4 \%)$ & $4(15 \%)$ & $5(19 \%)$ & $1(4 \% 0$ & $0(0 \%)$ & $11(41 \%)$ & $1(4 \%)$ & $2(7 \%)$ & $2(7 \%)$ \\
\hline & $\mathrm{P}$ value & \multicolumn{10}{|c|}{0.361} \\
\hline \multirow{5}{*}{ 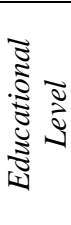 } & $\begin{array}{c}\text { Non formal } \\
\text { education }(n=23)\end{array}$ & $1(4 \%)$ & $0(0 \%)$ & $3(13 \%)$ & $3(13 \%)$ & $3(13 \%)$ & $0(0 \%)$ & $5(22 \%)$ & $0(0 \%)$ & $5(22 \%)$ & $3(13 \%)$ \\
\hline & Primary $(\mathrm{n}=48)$ & $1(2 \%)$ & $0(0 \%)$ & $9(19 \%)$ & $7(15 \%)$ & $5(10 \%)$ & $1(2 \%)$ & $9(19 \%)$ & $1(2 \%)$ & $11(23 \%)$ & $4(8 \%)$ \\
\hline & Secondary $(n=129)$ & $8(6 \%)$ & $1(1 \%)$ & $23(18 \%)$ & $20(16 \%)$ & $8(6 \%)$ & $1(1 \%)$ & $37(29 \%)$ & $1(1 \%)$ & $22(17 \%)$ & $8(6 \%)$ \\
\hline & Tertiary $(\mathrm{n}=47)$ & $1(2 \%)$ & $1(2 \%)$ & $13(28 \%)$ & $5(11 \%)$ & $9(19 \%)$ & $0(0 \%)$ & $8(17 \%)$ & $0(0 \%)$ & $9(19 \%)$ & $1(2 \%)$ \\
\hline & & \multicolumn{10}{|c|}{0.727} \\
\hline \multirow{8}{*}{ 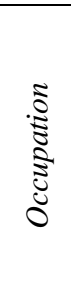 } & Trading $(n=101)$ & $5(5 \%)$ & $1(1 \%)$ & $25(25 \%)$ & $9(9 \%)$ & $9(9 \%)$ & $0(0 \%)$ & $28(28 \%)$ & $1(1 \%)$ & $15(15 \%)$ & $8(8 \%)$ \\
\hline & Farming $(n=3)$ & $0(0 \%)$ & $0(0 \%)$ & $1(33 \%)$ & $0(0 \%)$ & $1(33 \%)$ & $0(0 \%)$ & $0(0 \%)$ & $0(0 \%)$ & $1(33 \%)$ & $0(0 \%)$ \\
\hline & Driving $(\mathrm{n}=8)$ & $1(13 \%)$ & $0(0 \%)$ & $1(13 \%)$ & $3(38 \%)$ & $0(0 \%)$ & $0(0 \%)$ & $1(13 \%)$ & $0(0 \%)$ & $2(25 \%)$ & $0(0 \%)$ \\
\hline & Civil Service $(n=30)$ & $2(7 \%)$ & $1(3 \%)$ & $3(10 \%)$ & $5(17 \%)$ & $3(10 \%)$ & $0(0 \%)$ & $9(30 \%)$ & $1(3 \%)$ & $5(17 \%)$ & $1(3 \%)$ \\
\hline & Barbing $(\mathrm{n}=5)$ & $0(0 \%)$ & $0(0 \%)$ & $0(0 \%)$ & $1(20 \%)$ & $1(20 \%)$ & $0(0 \%)$ & $2(40 \%)$ & $0(0 \%)$ & $1(20 \%)$ & $0(0 \%)$ \\
\hline & Unemployed $(\mathrm{n}=36)$ & $1(3 \%)$ & $0(0 \%)$ & $4(11 \%)$ & $7(19 \%)$ & $5(14 \%)$ & $0(0 \%)$ & $10(28 \%)$ & $0(0 \%)$ & $6(17 \%)$ & $3(8 \%)$ \\
\hline & Others $(n=64)$ & $2(3 \%)$ & $0(0 \%)$ & $14(22 \%)$ & $10(16 \%)$ & $6(9 \% 0$ & $2(3 \%)$ & $9(14 \% 0$ & $0(0 \%)$ & $17(27 \%)$ & $4(6 \%)$ \\
\hline & $\mathrm{P}$ value & \multicolumn{10}{|c|}{0.918} \\
\hline \multirow{9}{*}{ 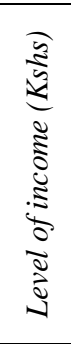 } & Less than $5,000(n=20)$ & $1(5 \%)$ & & & & $4(20 \%)$ & $0(0 \%)$ & $4(20 \%)$ & $0(0 \%)$ & $3(15 \%)$ & $1(5 \%)$ \\
\hline & $5,001-10,000(n=16)$ & $0(0 \%)$ & $1(6 \%)$ & $2(13 \%)$ & $2(13 \%)$ & $1(6 \%)$ & $0(0 \%)$ & $2(13 \%)$ & $0(0 \%)$ & $7(44 \%)$ & $1(6 \%)$ \\
\hline & $10,001-15,000(n=48)$ & $2(4 \%)$ & $0(0 \%)$ & $11(23 \%)$ & $7(15 \%)$ & $5(10 \%)$ & $0(0 \%)$ & $14(29 \%)$ & $0(0 \%)$ & $6(13 \%)$ & $3(6 \%)$ \\
\hline & $15,001-20,000(n=42)$ & $1(2 \%)$ & $0(0 \%)$ & $9(21 \%)$ & $9(21 \%)$ & $3(7 \%)$ & $0(0 \%)$ & $8(19 \%)$ & $1(2 \%)$ & $8(19 \%)$ & $3(7 \%)$ \\
\hline & $20,001-25,000(n=38)$ & $3(8 \%)$ & $1(3 \%)$ & $6(16 \%)$ & $3(8 \%)$ & $2(5 \%)$ & $1(3 \%)$ & $12(32 \%)$ & $1(3 \%)$ & $8(21 \%)$ & $1(3 \%)$ \\
\hline & $25,001-30,000(n=19)$ & $0(0 \%)$ & $0(0 \%)$ & $5(26 \%)$ & $3(16 \%)$ & $4(21 \%)$ & $0(0 \%)$ & $5(26 \%)$ & $0(0 \%)$ & $1(5 \%)$ & $1(5 \%)$ \\
\hline & $30,001-35,000(n=14)$ & $2(14 \%)$ & $0(0 \%)$ & $2(14 \%)$ & $4(29 \%)$ & $1(7 \%)$ & $0(0 \%)$ & $3(21 \%)$ & $0(0 \%)$ & $2(14 \%)$ & $0(0 \%)$ \\
\hline & $35,001-40,000(n=17)$ & $0(0 \%)$ & $0(0 \%)$ & $5(29 \%)$ & $0(0 \%)$ & $2(12 \%)$ & $1(6 \%)$ & $3(18 \%)$ & $0(0 \%)$ & $4(24 \%)$ & $2(12 \%)$ \\
\hline & $40,001-45,000(n=9)$ & $1(11 \%)$ & $0(0 \%)$ & $0(0 \%)$ & $1(11 \%)$ & $0(0 \%)$ & $0(0 \%)$ & $3(33 \%)$ & $0(0 \%)$ & $4(44 \%)$ & $0(0 \%)$ \\
\hline
\end{tabular}




\begin{tabular}{|c|c|c|c|c|c|c|c|c|c|c|}
\hline $45,001-50,000(n=13)$ & $0(0 \%)$ & $0(0 \%)$ & $3(23 \%)$ & $1(8 \%)$ & $2(15 \%)$ & $0(0 \%)$ & $4(31 \%)$ & $0(0 \%)$ & $2(15 \%)$ & $1(8 \%)$ \\
\hline More than $50,000(n=11)$ & $1(9 \%)$ & $0(0 \%)$ & $1(9 \%)$ & $2(18 \%)$ & $1(9 \%)$ & $0(0 \%)$ & $1(9 \%)$ & $0(0 \%)$ & $2(18 \%)$ & $3(27 \%)$ \\
\hline P value & \multicolumn{10}{|c|}{0.820} \\
\hline
\end{tabular}

Source: Servey, August 2017

The respondents were asked about the space where they grow crops. The farmers' responses were varied. The spaces are categorized into; rooftops, balconies, vacant places, in containers, along the railways, below power lines, river banks, school gardens, road strips and others to give us a better understanding on the choice of spaces for farming in Kibera. About $24 \%$ of the respondents planted along the river banks such as water lines and sewage lines and19\% on the road strips. There was considerable variation in the choice of space for farming between farmers from different age groups (Table 2(iii)). Thirty (30\%) of the older adults (>55 years), did farming on vacant places to $16 \%$ of young adults (<35 years). The choice of space also varied significantly amongst different gender groups $(\mathrm{p}=0.904)$, age groups $(\mathrm{p}=0.688)$, marital status $(\mathrm{p}=0.361)$, education level $(\mathrm{p}=0.727)$ occupation $(n=0.918)$ and level of income groups $(\mathrm{p}=0.820)$.

\subsection{Reasons/ Benefits for Adoption of Technology}

Table3(i). Frequency distribution on reasons for adoption of urban-technologies, according to socialdemographic groups in urban areas, Kasarani

\begin{tabular}{|c|c|c|c|c|c|c|c|}
\hline & $\begin{array}{l}\text { Socio-economic } \\
\text { characteristics }\end{array}$ & $\begin{array}{l}\text { Source } \\
\text { of food }\end{array}$ & $\begin{array}{c}\text { Source } \\
\text { of } \\
\text { income }\end{array}$ & $\begin{array}{c}\text { Unemploy } \\
\text { ment }\end{array}$ & $\begin{array}{c}\text { Use of } \\
\text { available } \\
\text { water and } \\
\quad \text { land }\end{array}$ & $\begin{array}{c}\text { High } \\
\text { dependency }\end{array}$ & Others \\
\hline \multirow{4}{*}{$\stackrel{\check{J}}{ら}$} & Male $(n=34)$ & $(20) 59 \%$ & $(7) 21 \%$ & (3)9\% & $(1) 3 \%$ & (2) $6 \%$ & (1)3\% \\
\hline & Female $(n=104)$ & $(81) 78 \%$ & (11)11\% & (2) $2 \%$ & (1)1\% & $(6) 6 \%$ & (3) $3 \%$ \\
\hline & Total $(n=138)$ & $(101) 73 \%$ & $(18) 13 \%$ & (5) $4 \%$ & $(2) 1 \%$ & $(8) 6 \%$ & (4) $3 \%$ \\
\hline & $\mathrm{P}$ value & \multicolumn{6}{|c|}{0.199} \\
\hline \multirow{4}{*}{$\stackrel{\infty}{\square}$} & Young adults $(\mathrm{n}=31)$ & (19)61\% & $(5) 16 \%$ & $(2) 7 \%$ & $(1) 3 \%$ & $(4) 13 \%$ & $(0) 0 \%$ \\
\hline & Middle aged adults $(\mathrm{n}=96)$ & $(73) 76 \%$ & (12)13\% & (3)3\% & (1)1\% & (4) $4 \%$ & (3)3\% \\
\hline & Older adults $(\mathrm{n}=11)$ & $(9) 82 \%$ & $(1) 9 \%$ & $(0) 0 \%$ & $(0) 0 \%$ & $(0) 0 \%$ & (1)9\% \\
\hline & $\mathrm{P}$ value & \multicolumn{6}{|c|}{0.34} \\
\hline \multirow{5}{*}{ 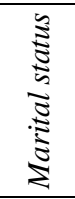 } & Single $(n=13)$ & $(9) 69 \%$ & (3)23\% & $(1) 8 \%$ & $(0) 0 \%$ & $(0) 0 \%$ & $(0) 0 \%$ \\
\hline & Married(n=98) & $(70) 71 \%$ & (12)12\% & (4) $4 \%$ & (2) $2 \%$ & $(8) 8 \%$ & (2) $2 \%$ \\
\hline & Divorced/separated $(n=4)$ & $(3) 75 \%$ & $(0) 0 \%$ & $(0) 0 \%$ & $(0) 0 \%$ & $(0) 0 \%$ & (1)25\% \\
\hline & Widowed $(\mathrm{n}=23)$ & (19)83\% & $(3) 13 \%$ & $(0) 0 \%$ & $(0) 0 \%$ & $(0) 0 \%$ & (1) $4 \%$ \\
\hline & P value & \multicolumn{6}{|c|}{0.439} \\
\hline \multirow{5}{*}{ 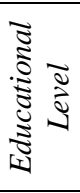 } & Non formal education $(n=8)$ & (6)75\% & (1)13\% & (0)0\% & (0)0\% & (0)0\% & (1)13\% \\
\hline & Primary $(n=16)$ & (13) $81 \%$ & (3)19\% & $(0) 0 \%$ & $(0) 0 \%$ & $(0) 0 \%$ & $(0) 0 \%$ \\
\hline & Secondary $(n=41)$ & $(32) 78 \%$ & $(2) 5 \%$ & $(2) 5 \%$ & (1) $2 \%$ & (4) $10 \%$ & $(0) 0 \%$ \\
\hline & Tertiary $(n=73)$ & $(50) 69 \%$ & (12)16\% & (3) $4 \%$ & $(1) 1 \%$ & $(4) 6 \%$ & (3) $4 \%$ \\
\hline & $\mathrm{P}$ value & \multicolumn{6}{|c|}{0.645} \\
\hline \multirow{8}{*}{$\begin{array}{l}\tilde{\vdots} \\
\bar{\Xi} \\
\vdots \\
0 \\
0 \\
0\end{array}$} & Trading $(n=48)$ & $(35) 73 \%$ & $(7) 15 \%$ & (1)2\% & $(1) 2 \%$ & (3) $6 \%$ & (1) $2 \%$ \\
\hline & Farming $(n=2)$ & $(0) 0 \%$ & $(0) 0 \%$ & $(1) 50 \%$ & $(0) 0 \%$ & $(0) 0 \%$ & $(1) 50 \%$ \\
\hline & Driving $(\mathrm{n}=3)$ & $(0) 0 \%$ & (2)67\% & $(0) 0 \%$ & $(0) 0 \%$ & (1) $33 \%$ & $(0) 0 \%$ \\
\hline & Civil Service $(\mathrm{n}=30)$ & $(22) 73 \%$ & (3) $10 \%$ & (1)3\% & (1)3\% & (3) $10 \%$ & $(0) 0 \%$ \\
\hline & Barbing $(\mathrm{n}=1)$ & (1) $100 \%$ & $(0) 0 \%$ & $(0) 0 \%$ & $(0) 0 \%$ & $(0) 0 \%$ & $(0) 0 \%$ \\
\hline & Unemployed(n=16) & $(13) 81 \%$ & $(0) 0 \%$ & $(2) 13 \%$ & $(0) 0 \%$ & $(0) 0 \%$ & $(1) 1 \%$ \\
\hline & Others $(\mathrm{n}=38)$ & $(30) 79 \%$ & $(6) 16 \%$ & $(0) 0 \%$ & $(0) 0 \%$ & $(1) 3 \%$ & (1)3\% \\
\hline & $\mathrm{P}$ value & \multicolumn{6}{|c|}{$\mathrm{P}<0.05$} \\
\hline \multirow{12}{*}{ 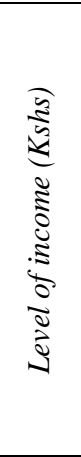 } & Less than $5,000(n=3)$ & (3) $100 \%$ & $(0) 0 \%$ & $(0) 0 \%$ & $(0) 0 \%$ & $(0) 0 \%$ & $(0) 0 \%$ \\
\hline & $5,001-10,000(\mathrm{n}=5)$ & $(2) 40 \%$ & $(3) 60 \%$ & $(0) 0 \%$ & $(0) 0 \%$ & $(0) 0 \%$ & $(0) 0 \%$ \\
\hline & $10,001-15,000(\mathrm{n}=18)$ & $(14) 78 \%$ & $(1) 6 \%$ & $(1) 6 \%$ & $(0) 0 \%$ & $(1) 6 \%$ & (1) $6 \%$ \\
\hline & $15,001-20,000(n=31)$ & $(22) 71 \%$ & $(4) 13 \%$ & (1)3\% & $(0) 0 \%$ & (3) $10 \%$ & (1)3\% \\
\hline & $20,001-25,000(\mathrm{n}=28)$ & $(24) 86 \%$ & $(2) 7 \%$ & $(0) 0 \%$ & $(0) 0 \%$ & $(2) 7 \%$ & $(0) 0 \%$ \\
\hline & $25,001-30,000(\mathrm{n}=19)$ & (11)58\% & (3) $16 \%$ & (1)5\% & (2)11\% & $(1) 5 \%$ & (1)5\% \\
\hline & $30,001-35,000(\mathrm{n}=13)$ & (12)92\% & $(0) 0 \%$ & $(1) 8 \%$ & $(0) 0 \%$ & $(0) 0 \%$ & $(0) 0 \%$ \\
\hline & $35,001-40,000(n=9)$ & $(6) 67 \%$ & $(0) 0 \%$ & (1)11\% & $(0) 0 \%$ & $(1) 11 \%$ & (1) $11 \%$ \\
\hline & $40,001-45,000(n=5)$ & (3) $60 \%$ & (2) $40 \%$ & $(0) 0 \%$ & $(0) 0 \%$ & $(0) 0 \%$ & $(0) 0 \%$ \\
\hline & $45,001-50,000(n=4)$ & $(2) 50 \%$ & $(2) 50 \%$ & $(0) 0 \%$ & $(0) 0 \%$ & $(0) 0 \%$ & $(0) 0 \%$ \\
\hline & More than $50,000(n=3)$ & (2) $67 \%$ & (1)33\% & $(0) 0 \%$ & $(0) 0 \%$ & $(0) 0 \%$ & $(0) 0 \%$ \\
\hline & $\mathrm{P}$ value & \multicolumn{6}{|c|}{0.503} \\
\hline
\end{tabular}

$* \mathrm{p}<0.05$

Source: survey, August, 2017 
In the analysis of the main reasons for adoption of urban horticultural technologies, the farmers' responses were varied in Kasarani. They are categorized into a source of food, source of income, unemployment, use of available water and land and high dependence. About $73 \%$ respondents said the source of food is the main reason for adoption of urban horticultural technologies, followed by 'source of income' (13\%), and about 6\% and 4\% indicated high dependency and unemployment respectively (Table 3 i). Reason for adoption varied significantly amongst different social demographic groups, sex groups $(\mathrm{p}=0.199)$, age groups $(\mathrm{p}=0.34)$, and occupation groups $(\mathrm{p}<0.05)$.

Table3(ii). Frequency distribution on reasons for adoption of urban-technologies, according to socialdemographic groups in urban areas, Mathare

\begin{tabular}{|c|c|c|c|c|c|c|c|}
\hline & $\begin{array}{c}\text { Socio-economic } \\
\text { characteristics }\end{array}$ & $\begin{array}{c}\text { Source of } \\
\text { food }\end{array}$ & $\begin{array}{c}\begin{array}{c}\text { Source of } \\
\text { income }\end{array} \\
\end{array}$ & Unemployment & $\begin{array}{c}\text { Use of available } \\
\text { water and land }\end{array}$ & $\begin{array}{c}\text { High } \\
\text { dependency }\end{array}$ & Others \\
\hline \multirow{4}{*}{ ¿ } & Male $(\mathrm{n}=61)$ & $48(79 \%)$ & $5(8 \%)$ & $5(8 \%)$ & $1(2 \%)$ & $0(0 \%)$ & $2(3 \%)$ \\
\hline & Female $(n=134)$ & $101(75 \%)$ & $19(14 \%)$ & $2(2 \%)$ & $4(3 \%)$ & $3(2 \%)$ & $5(4 \%)$ \\
\hline & Total(n=195) & $149(76 \%)$ & $24(12 \%)$ & $7(4 \%)$ & $5(3 \%)$ & $3(2 \%)$ & $7(4 \%)$ \\
\hline & $\mathrm{P}$ value & \multicolumn{6}{|c|}{0.145} \\
\hline \multirow{5}{*}{$\stackrel{\infty}{\infty}$} & Young adults(n=49) & $33(67 \%)$ & $9(18 \%)$ & $2(4 \%)$ & $4(8 \%)$ & $0(0 \%)$ & $1(2 \%)$ \\
\hline & Middle aged adults $(n=132)$ & $104(49 \%)$ & $13(10 \%)$ & $5(4 \%)$ & $1(1 \%)$ & $3(2 \%)$ & $6(5 \%)$ \\
\hline & Older adults $(\mathrm{n}=14)$ & $12(86 \%)$ & $2(14 \%)$ & $0(0 \%)$ & $0(0 \%)$ & $0(0 \%)$ & $0(0 \%)$ \\
\hline & Total $(n=195)$ & $149(76 \%)$ & $24(12 \%)$ & $7(4 \%)$ & $5(3 \%)$ & $3(2 \%)$ & $7(4 \%)$ \\
\hline & $\mathrm{P}$ value & \multicolumn{6}{|c|}{0.043} \\
\hline \multirow{5}{*}{ 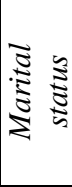 } & Single $(n=22)$ & $17(77 \%)$ & $5(23 \%)$ & $0(0 \%)$ & $0(0 \%)$ & $0(0 \%)$ & $0(0 \%)$ \\
\hline & Married(n=144) & $109(76 \%)$ & $16(11 \%)$ & $5(4 \%)$ & $4(3 \%)$ & $3(2 \%)$ & $7(5 \%)$ \\
\hline & Divorced/separated(n=10) & $9(90 \%)$ & $0(0 \%)$ & $1(10 \%)$ & $0(0 \%)$ & $0(0 \%)$ & $0(0 \%)$ \\
\hline & Widowed(n=19) & $14(74 \%)$ & $3(16 \%)$ & $1(5 \%)$ & $1(5 \%)$ & $0(0 \%)$ & $0(0 \%)$ \\
\hline & $\mathrm{P}$ value & \multicolumn{6}{|c|}{0.766} \\
\hline \multirow{5}{*}{ 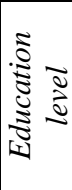 } & Non formal education $(n=16)$ & $12(75 \%)$ & $3(19 \%)$ & $1(6 \%)$ & $2(13 \%)$ & $1(6 \%)$ & $2(13 \%)$ \\
\hline & $\operatorname{Primary}(\mathrm{n}=26)$ & $20(77 \%)$ & $6(23 \%)$ & $2(7 \%)$ & $1(4 \%)$ & $0(0 \%)$ & $1(4 \%)$ \\
\hline & Secondary $(\mathrm{n}=108)$ & $85(79 \%)$ & $11(10 \%)$ & $4(4 \%)$ & $1(1 \%)$ & $0(0 \%)$ & $3(3 \%)$ \\
\hline & Tertiary $(n=45)$ & $32(71 \%)$ & $4(9 \%)$ & $0(0 \%)$ & $1(2 \%)$ & $2(4 \%)$ & $1(2 \%)$ \\
\hline & $\mathrm{P}$ value & \multicolumn{6}{|c|}{0.617} \\
\hline \multirow{8}{*}{ 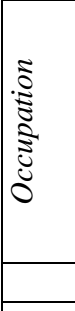 } & Trading $(\mathrm{n}=87)$ & $63(72 \%)$ & $13(15 \%)$ & $2(2 \%)$ & $2(2 \%)$ & $2(2 \%)$ & $5(6 \%)$ \\
\hline & Farming $(n=4)$ & $4(100 \%)$ & $0(0 \%)$ & $0(0 \%)$ & $0(0 \%)$ & $0(0 \%)$ & $0(0 \%)$ \\
\hline & Driving $(\mathrm{n}=4)$ & $3(75 \%)$ & $1(25 \%)$ & $0(0 \%)$ & $0(0 \%)$ & $0(0 \%)$ & $0(0 \%)$ \\
\hline & Civil Service $(\mathrm{n}=25)$ & $16(64 \%)$ & $5(20 \%)$ & $1(4 \%)$ & $2(8 \%)$ & $0(0 \%)$ & $1(4 \%)$ \\
\hline & Barbing $(\mathrm{n}=2)$ & $1(50 \%)$ & $1(50 \%)$ & $0(0 \%)$ & $0(0 \%)$ & $0(0 \%)$ & $0(0 \%)$ \\
\hline & Unemployed $(\mathrm{n}=28)$ & $24(86 \%)$ & $2(7 \%)$ & $1(4 \%)$ & $0(0 \%)$ & $0(0 \%)$ & $1(4 \%)$ \\
\hline & Others $(n=45)$ & $38(84 \%)$ & $2(4 \%)$ & $3(7 \%)$ & $1(2 \%)$ & $1(2 \%)$ & $0(0 \%)$ \\
\hline & $\mathrm{P}$ value & \multicolumn{6}{|c|}{0.984} \\
\hline \multirow{12}{*}{ 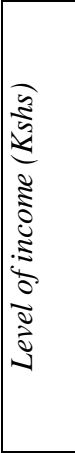 } & Less than $5,000(n=11)$ & $8(73 \%)$ & $2(18 \%)$ & $0(0 \%)$ & $1(9 \%)$ & $0(0 \%)$ & $0(0 \%)$ \\
\hline & $5,001-10,000(\mathrm{n}=23)$ & $17(74 \%)$ & $2(9 \%)$ & $0(0 \%)$ & $2(9 \%)$ & $0(0 \%)$ & $2(9 \%)$ \\
\hline & $10,001-15,000(n=41)$ & $34(83 \%)$ & $5(12 \%)$ & $1(2 \%)$ & $0(0 \%)$ & $0(0 \%)$ & $1(2 \%)$ \\
\hline & $15,001-20,000(n=39)$ & $31(80 \%)$ & $3(8 \%)$ & $2(5 \%)$ & $1(3 \%)$ & $0(0 \%)$ & $2(5 \%)$ \\
\hline & $20,001-25,000(n=19)$ & $16(84 \%)$ & $2(11 \%)$ & $1(5 \%)$ & $0(0 \%)$ & $0(0 \%)$ & $0(0 \%)$ \\
\hline & $25,001-30,000(n=12)$ & $10(8 \%)$ & $2(17 \%)$ & $0(0 \%)$ & $0(0 \%)$ & $0(0 \%)$ & $0(0 \%)$ \\
\hline & $30,001-35,000(n=16)$ & $10(6 \%)$ & $4(25 \%)$ & $0(0 \%)$ & $1(6 \%)$ & $0(0 \%)$ & $1(6 \%)$ \\
\hline & $35,001-40,000(n=14)$ & $10(7 \%)$ & $1(7 \%)$ & $1(7 \%)$ & $0(0 \%)$ & $1(7 \%)$ & $1(7 \%)$ \\
\hline & $40,001-45,000(n=6)$ & $4(67 \%)$ & $1(17 \%)$ & $1(17 \%)$ & $0(0 \%)$ & $0(0 \%)$ & $0(0 \%)$ \\
\hline & $45,001-50,000(\mathrm{n}=8)$ & $6(75 \%)$ & $2(25 \%)$ & $0(0 \%)$ & $0(0 \%)$ & $0(0 \%)$ & $0(0 \%)$ \\
\hline & More than $50,000(n=6)$ & $3(50 \%)$ & $0(0 \%)$ & $1(17 \%)$ & $0(0 \%)$ & $2(33 \%)$ & $0(0 \%)$ \\
\hline & $\mathrm{P}$ value & \multicolumn{6}{|c|}{0.011} \\
\hline
\end{tabular}

${ }^{*} \mathrm{p}<0.05$

Source: Survey, August 2017

The analysis of the main reasons for adoption of urban horticultural technologies showed that the farmers' responses were varied in Mathare. They are categorized into a source of food, source of income, unemployment, use of available water and land and high dependence. About $76 \%$ respondents said the source of food as the main reason for adoption of urban horticultural technologies, followed by 'source of income' (12\%), and about $7 \%$ indicated unemployment (Table 3 ii). Reason for adoption varied significantly amongst different social demographic groups, sex groups $(\mathrm{p}=0.145)$, age groups $(\mathrm{p}=0.043)$, and level of income $(\mathrm{p}=0.011)$. 
Table3(iii). Frequency distribution on reasons for adoption of urban-technologies, according to socialdemographic groups in urban areas, Kibera

\begin{tabular}{|c|c|c|c|c|c|c|c|}
\hline & $\begin{array}{l}\text { Socio-economic } \\
\text { characteristics }\end{array}$ & $\begin{array}{l}\text { Source } \\
\text { of food }\end{array}$ & $\begin{array}{c}\text { Source } \\
\text { of income }\end{array}$ & Unemployment & $\begin{array}{c}\text { Use of available } \\
\text { water and land }\end{array}$ & $\begin{array}{c}\text { High } \\
\text { dependency }\end{array}$ & Others \\
\hline \multirow{4}{*}{ ๘ँ } & Male(n=74) & $52(70 \%)$ & $9(12 \%)$ & $4(5 \%)$ & $1(1 \%)$ & $1(1 \%)$ & $7(10 \%)$ \\
\hline & Female $(n=173)$ & $123(71 \%)$ & $28(16 \%)$ & $6(4 \%)$ & $4(2 \%)$ & $5(3 \%)$ & $7(4 \%)$ \\
\hline & Total $(n=247)$ & $175(71 \%)$ & $37(15 \%)$ & $10(4 \%)$ & $5(2 \%)$ & $6(2 \%)$ & $14(6 \%)$ \\
\hline & $\mathrm{P}$ value & \multicolumn{6}{|c|}{0.484} \\
\hline \multirow{5}{*}{$\infty$} & Young adults(n=64) & $37(58 \%)$ & $17(7 \%)$ & $2(3 \%)$ & $3(5 \%)$ & $1(2 \%)$ & $4(6 \%)$ \\
\hline & Middle aged $(\mathrm{n}=160)$ & $120(75 \%)$ & $18(11 \%)$ & $8(5 \%)$ & $2(1 \%)$ & $4(3 \%)$ & $8(5 \%)$ \\
\hline & Older adults $(\mathrm{n}=23)$ & $18(78 \%)$ & $2(9 \%)$ & $0(0 \%)$ & $0(0 \%)$ & $1(4 \%)$ & $2(9 \%)$ \\
\hline & $\operatorname{Total}(\mathrm{n}=247)$ & $175(71 \%)$ & $37(15 \%)$ & $10(4 \%)$ & $5(2 \%)$ & $6(2 \%)$ & $14(6 \%)$ \\
\hline & $\mathrm{P}$ value & \multicolumn{6}{|c|}{0.499} \\
\hline \multirow{5}{*}{ 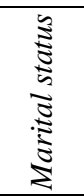 } & Single $(n=40)$ & $30(75 \%)$ & $5(13 \%)$ & $1(3 \%)$ & $2(5 \%)$ & $1(3 \%)$ & $1(3 \%)$ \\
\hline & Married(n=170) & $115(68 \%)$ & $28(17 \%)$ & $9(5 \%)$ & $3(2 \%)$ & $3(2 \%)$ & $12(7 \%)$ \\
\hline & Divorced/separated $(\mathrm{n}=10)$ & $9(90 \%)$ & $1(10 \%)$ & $0(0 \%)$ & $0(0 \%)$ & $0(0 \%)$ & $0(0 \%)$ \\
\hline & Windowed $(\mathrm{n}=27)$ & $21(78 \%)$ & $3(11 \%)$ & $0(0 \%)$ & $0(0 \%)$ & $2(7 \%)$ & $1(4 \%)$ \\
\hline & $\mathrm{P}$ value & \multicolumn{6}{|c|}{0.665} \\
\hline \multirow{5}{*}{ 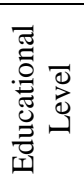 } & Non formal education $(n=23)$ & $16(70 \%)$ & $4(17 \%)$ & $0(0 \%)$ & $0(0 \%)$ & $1(4 \%)$ & $2(9 \%)$ \\
\hline & Primary $(\mathrm{n}=48)$ & $34(71 \%)$ & $9(19 \%)$ & $1(2 \%)$ & $2(4 \%)$ & $0(0 \%)$ & $2(4 \%)$ \\
\hline & Secondary $(n=129)$ & $96(74 \%)$ & $16(12 \%)$ & $6(5 \%)$ & $1(1 \%)$ & $4(3 \%)$ & $6(5 \%)$ \\
\hline & $\operatorname{Tertiary}(n=47)$ & $29(62 \%)$ & $8(17 \%)$ & $3(6 \%)$ & $2(4 \%)$ & $1(2 \%)$ & $4(9 \%)$ \\
\hline & $\mathrm{P}$ value & \multicolumn{6}{|c|}{0.744} \\
\hline \multirow{7}{*}{ 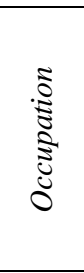 } & Trading $(\mathrm{n}=101)$ & $72(71 \%)$ & $15(15 \%)$ & $4(4 \%)$ & $2(2 \%)$ & $3(3 \%)$ & $5(5 \%)$ \\
\hline & Farming $(\mathrm{n}=3)$ & $2(67 \%)$ & $1(33 \%)$ & $2(67 \%)$ & $0(0 \%)$ & $0(0 \%)$ & $0(0 \%)$ \\
\hline & Driving $(\mathrm{n}=8)$ & $7(88 \%)$ & $0(0 \%)$ & $0(0 \%)$ & $1(13 \%)$ & $0(0 \%)$ & $0(0 \%)$ \\
\hline & Civil Service $(\mathrm{n}=30)$ & $18(60 \%)$ & $6(20 \%)$ & $1(3 \%)$ & $0(0 \%)$ & $0(0 \%)$ & $5(17 \%)$ \\
\hline & Barbing $(n=5)$ & $4(80 \%)$ & $1(20 \%)$ & $0(0 \%)$ & $0(0 \%)$ & $0(0 \%)$ & $0(0 \%)$ \\
\hline & Unemployed $(\mathrm{n}=36)$ & $22(61 \%)$ & $7(19 \%)$ & $3(8 \%)$ & $1(3 \%)$ & $2(6 \%)$ & $1(3 \%)$ \\
\hline & Others $(\mathrm{n}=64)$ & $51(80 \%)$ & $6(9 \%)$ & $2(3 \%)$ & $1(2 \%)$ & $1(2 \%)$ & $3(5 \%)$ \\
\hline & P value & \multicolumn{6}{|c|}{0.197} \\
\hline \multirow{12}{*}{ 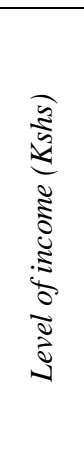 } & Less than $5,000(n=20)$ & $15(75 \%)$ & $3(15 \%)$ & $0(0 \%)$ & $1(5 \%)$ & $1(5 \%)$ & $0(0 \%)$ \\
\hline & $5,001-10,000(n=16)$ & $10(63 \%)$ & $3(19 \%)$ & $0(0 \%)$ & $1(6 \%)$ & $0(0 \%)$ & $2(13 \%)$ \\
\hline & $10,001-15,000(\mathrm{n}=48)$ & $33(69 \%)$ & $9(19 \%)$ & $4(8 \%)$ & $0(0 \%)$ & $0(0 \%)$ & $2(4 \%)$ \\
\hline & $15,001-20,000(n=42)$ & $35(83 \%)$ & $4(10 \%)$ & $2(5 \%)$ & $0(0 \%)$ & $0(0 \%)$ & $1(2 \%)$ \\
\hline & $20,001-25,000(\mathrm{n}=38)$ & $27(71 \%)$ & $5(13 \%)$ & $0(0 \%)$ & $1(3 \%)$ & $2(5 \%)$ & $3(8 \%)$ \\
\hline & $25,001-30,000(\mathrm{n}=19)$ & $13(68 \%)$ & $4(21 \%)$ & $0(0 \%)$ & $0(0 \%)$ & $1(5 \%)$ & $1(5 \%)$ \\
\hline & $30,001-35,000(n=14)$ & $7(50 \%)$ & $1(7 \%)$ & $0(0 \%)$ & $1(7 \%)$ & $1(7 \%)$ & $4(29 \%)$ \\
\hline & $35,001-40,000(n=17)$ & $12(71 \%)$ & $1(6 \%)$ & $3(18 \%)$ & $0(0 \%)$ & $0(0 \%)$ & $1(6 \%)$ \\
\hline & $40,001-45,000(n=9)$ & $4(45 \%)$ & $4(45 \%)$ & $0(0 \%)$ & $0(0 \%)$ & $1(11 \%)$ & $0(0 \%)$ \\
\hline & $45,001-50,000(n=13)$ & $10(77 \%)$ & $2(15 \%)$ & $0(0 \%)$ & $1(8 \%)$ & $0(0 \%)$ & $0(0 \%)$ \\
\hline & More than $50,000(n=11)$ & $9(82 \%)$ & $1(9 \%)$ & $1(9 \%)$ & $0(0 \%)$ & $0(0 \%)$ & $0(0 \%)$ \\
\hline & $\mathrm{P}$ value & \multicolumn{6}{|c|}{0.065} \\
\hline
\end{tabular}

*p $<0.05$

Source: Survey, August 2017

The analysis of the main reasons for adoption of urban horticultural technologies showed that the farmers' responses were varied. They are categorized into a source of food, source of income, unemployment, use of available water and land and high dependence. About $71 \%$ of the respondents said the source of food is the main reason for adoption of urban horticultural technologies, followed by 'source of income' 15\%, and about $4 \%$ indicated unemployment (Table 3 iii). Reason for adoption varied significantly amongst different social demographic groups, sex groups $(\mathrm{p}=0.484)$, age groups $(\mathrm{p}=0.499)$, and level of income $(\mathrm{p}=0.065)$.

\subsection{Challenges Farmers Faced in Adoption of Urban Farming Technologies}

Table4. Frequency distribution on challengesfarmers faced in adopting of urban technologies according to social-demographic groups, Kibera

\begin{tabular}{|l|l|l|l|l|l|l|l|l|}
\hline & $\begin{array}{l}\text { Socio-economic } \\
\text { characteristics }\end{array}$ & $\begin{array}{l}\text { Pests \& } \\
\text { Diseases }\end{array}$ & $\begin{array}{l}\text { Inadequate } \\
\text { capital }\end{array}$ & $\begin{array}{l}\text { Slashing } \\
\text { crops }\end{array}$ & security & $\begin{array}{l}\text { Inadequate } \\
\text { inputs }\end{array}$ & $\begin{array}{l}\text { Inadequate } \\
\text { Market }\end{array}$ & $\begin{array}{l}\text { Any } \\
\text { other }\end{array}$ \\
\hline \multirow{2}{*}{$๘$} & Male(n=74) & $18(24 \%)$ & $6(8 \%)$ & $6(8 \%)$ & $46(62 \%)$ & $0(0 \%)$ & $0(0 \%)$ & $4(5 \%)$ \\
\cline { 2 - 8 } & Female(n=173) & $0(0 \%)$ & $17(10 \%)$ & $34(20 \%)$ & $83(48 \%)$ & $20(12 \%)$ & $4(2 \%)$ & $25(15 \%)$ \\
\cline { 2 - 8 } & Total(n=247) & $18(7 \%)$ & $23(9 \%)$ & $40(16 \%)$ & $129(52 \%)$ & $20(8 \%)$ & $4(2 \%)$ & $29(12 \%)$ \\
\hline \multirow{2}{*}{$\odot$} & Young adults(n=64) & $7(11 \%)$ & $9(14 \%)$ & $8(13 \%)$ & $20(31 \%)$ & $5(8 \%)$ & $2(3 \%)$ & $13(20 \%)$ \\
\hline
\end{tabular}




\begin{tabular}{|c|c|c|c|c|c|c|c|c|}
\hline & Middle aged $(n=160)$ & $5(3 \%)$ & $7(4 \%)$ & $25(16 \%)$ & $91(57 \%)$ & $15(9 \%)$ & $2(1 \%)$ & $15(9 \%)$ \\
\hline & Older adults $(\mathrm{n}=23)$ & $6(26 \%)$ & $7(30 \%)$ & $7(30 \%)$ & $18(78 \%)$ & $0(0 \%)$ & $0(0 \%)$ & $1(4 \%)$ \\
\hline \multirow{4}{*}{ 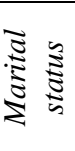 } & Single $(n=40)$ & $4(10 \%)$ & $4(10 \%)$ & $1(3 \%)$ & $31(78 \%)$ & $2(5 \%)$ & $0(0 \%)$ & $1(3 \%)$ \\
\hline & Married(n=170) & $13(8 \%)$ & $10(6 \%)$ & $33(19 \%)$ & $79(47 \%)$ & $16(9 \%)$ & $4(2 \%)$ & $25(15 \%)$ \\
\hline & Divorced/separated $(\mathrm{n}=10)$ & $1(10 \%)$ & $5(50 \%)$ & $0(0 \%)$ & $4(40 \%)$ & $0(0 \%)$ & $0(0 \%)$ & $0(0 \%)$ \\
\hline & Widowed(n=27) & $0(0 \%)$ & $4(15 \%)$ & $6(22 \%)$ & $15(55 \%)$ & $2(7 \%)$ & $0(0 \%)$ & $3(11 \%)$ \\
\hline \multirow{4}{*}{ 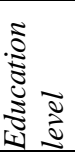 } & Non formal education $(n=23)$ & $6(26 \%)$ & $10(44 \%)$ & $1(4 \%)$ & $10(44 \%)$ & $1(4 \%)$ & $0(0 \%)$ & $0(0 \%)$ \\
\hline & Primary $(\mathrm{n}=48)$ & $1(2 \%)$ & $5(10 \%)$ & $7(15 \%)$ & $19(40 \%)$ & $3(6 \%)$ & $2(4 \%)$ & $12(25 \%)$ \\
\hline & $\operatorname{Secondary}(n=129)$ & $5(4 \%)$ & $7(5 \%)$ & $25(19 \%)$ & $69(54 \%)$ & $10(8 \%)$ & $2(2 \%)$ & $15(12 \%)$ \\
\hline & Tertiary $(n=47)$ & $6(13 \%)$ & $1(2 \%)$ & $7(15 \%)$ & $29(62 \%)$ & $6(13 \%)$ & $0(0 \%)$ & $2(4 \%)$ \\
\hline \multirow{7}{*}{ 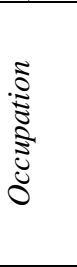 } & Trading $(\mathrm{n}=101)$ & $7(7 \%)$ & $7(7 \%)$ & $17(17 \%)$ & $57(56 \%)$ & $8(8 \%)$ & $1(1 \%)$ & $15(15 \%)$ \\
\hline & Farming $(\mathrm{n}=3)$ & $1(33 \%)$ & $0(0 \%)$ & $0(0 \%)$ & $1(33 \%)$ & $0(0 \%)$ & $0(0 \%)$ & $3(100 \%)$ \\
\hline & Driving $(\mathrm{n}=8)$ & $1(13 \%)$ & $0(0 \%)$ & $2(25 \%)$ & $3(38 \%)$ & $0(0 \%)$ & $0(0 \%)$ & $3(38 \%)$ \\
\hline & Civil Service $(\mathrm{n}=30)$ & $1(3 \%)$ & $3(10 \%)$ & $5(17 \%)$ & $17(57 \%)$ & $1(3 \%)$ & $1(3 \%)$ & $2(7 \%)$ \\
\hline & Barbing $(\mathrm{n}=5)$ & $0(0 \%)$ & $2(40 \%)$ & $1(20 \%)$ & $1(20 \%)$ & $0(0 \%)$ & $0(0 \%)$ & $1(20 \%)$ \\
\hline & Unemployed $(n=36)$ & $0(0 \%)$ & $7(19 \%)$ & $4(11 \%)$ & $18(50 \%)$ & $4(11 \%)$ & $1(3 \%)$ & $4(11 \%)$ \\
\hline & Others $(n=64)$ & $8(13 \%)$ & $6(9 \%)$ & $11(17 \%)$ & $32(50 \%)$ & $7(11 \%)$ & $1(2 \%)$ & $1(2 \%)$ \\
\hline \multirow{11}{*}{ 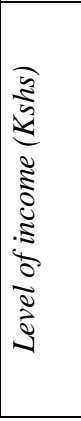 } & Less than $5,000(n=20)$ & $3(15 \%)$ & $0(0 \%)$ & $0(0 \%)$ & $18(90 \%)$ & $0(0 \%)$ & $0(0 \%)$ & $0(0 \%)$ \\
\hline & $5,001-10,000(n=16)$ & $1(6 \%)$ & $1(6 \%)$ & $1(6 \%)$ & $7(44 \%)$ & $3(19 \%)$ & $0(0 \%)$ & $3(19 \%)$ \\
\hline & $10,001-15,000(\mathrm{n}=51)$ & $3(6 \%)$ & $6(12 \%)$ & $11(22 \%)$ & $21(41 \%)$ & $4(8 \%)$ & $0(0 \%)$ & $6(12 \%)$ \\
\hline & $15,001-20,000(n=42)$ & $0(0 \%)$ & $2(5 \%)$ & $7(17 \%)$ & $23(55 \%)$ & $3(7 \%)$ & $3(7 \%)$ & $2(5 \%)$ \\
\hline & $20,001-25,000(n=47)$ & $4(9 \%)$ & $6(13 \%)$ & $6(13 \%)$ & $20(43 \%)$ & $2(4 \%)$ & $0(0 \%)$ & $9(19 \%)$ \\
\hline & $25,001-30,000(n=20)$ & $1(5 \%)$ & $0(0 \%)$ & $4(20 \%)$ & $10(50 \%)$ & $2(10 \%)$ & $0(0 \%)$ & $2(10 \%)$ \\
\hline & $30,001-35,000(n=15)$ & $1(7 \%)$ & $2(13 \%)$ & $3(20 \%)$ & $5(33 \%)$ & $1(7 \%)$ & $0(0 \%)$ & $1(7 \%)$ \\
\hline & $35,001-40,000(n=17)$ & $3(18 \%)$ & $2(12 \%)$ & $3(18 \%)$ & $11(65 \%)$ & $0(0 \%)$ & $0(0 \%)$ & $0(0 \%)$ \\
\hline & $40,001-45,000(\mathrm{n}=9)$ & $0(0 \%)$ & $1(11 \%)$ & $3(33 \%)$ & $4(44 \%)$ & $2(22 \%)$ & $0(0 \%)$ & $1(11 \%)$ \\
\hline & $45,001-50,000(n=13)$ & $2(15 \%)$ & $1(8 \%)$ & $0(0 \%)$ & $5(39 \%)$ & $3(23 \%)$ & $1(8 \%)$ & $1(8 \%)$ \\
\hline & More than $50,000(n=11)$ & $0(0 \%)$ & $2(18 \%)$ & $2(18 \%)$ & $5(46 \%)$ & $0(0 \%)$ & $0(0 \%)$ & $1(9 \%)$ \\
\hline
\end{tabular}

N:B These responses may not add to $100 \%$, because some respondents gave more than one response(multiple response)

Source: Survey, August 2017

Data on table 4 shows that $52 \%$ of the respondents felt that security of land ownership and theft of crops were the most severe challenges for urban horticulture. Other challenges are slashing of crops $(16 \%)$, pest and diseases $(7 \%)$, while only $2 \%$ mentioned inadequate market for vegetables. Those, whose level of income was less than 5,000, indicated that security and pests as well as diseases as the main challenges at $18 \%$ and $15 \%$ respectively.

\subsection{Kind of Vegetables Grown}

Table 5. Distribution frequency of kind of vegetables grown according to social-demographic groups, Mathare

\begin{tabular}{|c|c|c|c|c|c|c|c|c|c|}
\hline & $\begin{array}{l}\text { Socio-economic } \\
\text { characteristics }\end{array}$ & Kales & Spinach & Onions & Amaranth & Pumpkin & $\begin{array}{l}\text { Green } \\
\text { beans }\end{array}$ & Pepper & others \\
\hline \multirow{3}{*}{ ๘ँ } & Male(n=61) & $61(100 \%)$ & $35(57 \%)$ & $16(26 \%)$ & $30(49 \%)$ & $0(0 \%)$ & $0(0 \%)$ & $4(7 \%)$ & $6(10 \%)$ \\
\hline & Female $(\mathrm{n}=134)$ & $96(72 \%)$ & $80(60 \%)$ & $33(25 \%)$ & $24(18 \%)$ & $41(31 \%)$ & $12(9 \%)$ & $5(4 \%)$ & $15(11 \%)$ \\
\hline & $\operatorname{Total}(\mathrm{n}=195)$ & $157(81 \%)$ & $115(59 \%)$ & $49(25 \%)$ & $54(28 \%)$ & $41(21 \%)$ & $12(6 \%)$ & $9(5 \%)$ & $21(11 \%)$ \\
\hline \multirow{3}{*}{$\stackrel{\infty}{\infty}$} & Young adults $(\mathrm{n}=49)$ & $49(100 \%)$ & $29(59 \%)$ & $10(20 \%)$ & $12(24 \%)$ & $0(0 \%)$ & $0(0 \%)$ & $4(8 \%)$ & $9(18 \%)$ \\
\hline & Middle aged adults $(\mathrm{n}=132)$ & $106(80 \%)$ & $74(56 \%)$ & $39(30 \%)$ & $36(27 \%)$ & $41(31 \%)$ & $12(9 \%)$ & $5(4 \%)$ & $8(6 \%)$ \\
\hline & Older adults $(\mathrm{n}=14)$ & $2(14 \%)$ & $12(86 \%)$ & $0(0 \%)$ & $6(43 \%)$ & $0(0 \%)$ & $0(0 \%)$ & $0(0 \%)$ & $4(29 \%)$ \\
\hline \multirow{4}{*}{ 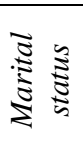 } & Single $(n=22)$ & $22(100 \%)$ & $12(55 \%)$ & $6(27 \%)$ & $13(59 \%)$ & $0(0 \%)$ & $0(0 \%)$ & $3(14 \%)$ & $2(14 \%)$ \\
\hline & Married(n=144) & $124(86 \%)$ & $82(57 \%)$ & $42(29 \%)$ & $32(22 \%)$ & $41(29 \%)$ & $12(8 \%)$ & $6(4 \%)$ & $11(8 \%)$ \\
\hline & Divorced/se & $6(60 \%)$ & $6(60 \%)$ & $0(0 \%)$ & $\%)$ & $0(0 \%)$ & $0(0 \%)$ & $0(0 \%)$ & $5(50 \%)$ \\
\hline & Widowed(r & $5(26 \%)$ & $15(79 \%)$ & $1(5 \%)$ & $6(32 \%)$ & $0(0 \%)$ & $0(0 \%)$ & $0(0 \%)$ & $3(16 \%)$ \\
\hline \multirow{7}{*}{ 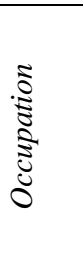 } & Trading $(\mathrm{n}=87)$ & $77(86 \%)$ & $48(55 \%)$ & $32(37 \%)$ & $37(43 \%)$ & $2(2 \%)$ & $0(0 \%)$ & $7(8 \%)$ & $19(22 \%)$ \\
\hline & Farming $(n=4)$ & $2(50 \%)$ & $1(25 \%)$ & $1(25 \%)$ & $2(50 \%)$ & $1(25 \%)$ & $0(0 \%)$ & $0(0 \%)$ & $0(0 \%)$ \\
\hline & Driving $(n=4)$ & $2(50 \%)$ & $1(25 \%)$ & $1(25 \%)$ & $2(50 \%)$ & $2(50 \%)$ & $0(0 \%)$ & $0(0 \%)$ & $0(0 \%)$ \\
\hline & Civil Se & $20(80 \%)$ & $18(7$ & $3(12 \%)$ & $4(16 \%)$ & $11(44 \%)$ & $0(0 \%)$ & $2(8 \%)$ & $1(4 \%)$ \\
\hline & $\operatorname{Barbing}(\mathrm{n}=2)$ & $2(100 \%)$ & $1(50 \%)$ & $1(50 \%)$ & $0(0 \%)$ & $1(50 \%)$ & $0(0 \%)$ & $0(0 \%)$ & $0(0 \%)$ \\
\hline & Unemployment $(\mathrm{n}=28)$ & $28(100 \%)$ & $17(61 \%)$ & $7(25 \%)$ & $1(4 \%)$ & $13(46 \%)$ & $6(21 \%)$ & $0(0 \%)$ & $0(0 \%)$ \\
\hline & Others $(n=45)$ & $26(58 \%)$ & $29(64 \%)$ & $4(9 \%)$ & $8(18 \%)$ & $11(24 \%)$ & $6(13 \%)$ & $0(0 \%)$ & $17(38 \%)$ \\
\hline \multirow{4}{*}{ 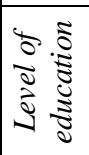 } & Non formal education $(n=16)$ & $16(100 \%)$ & $10(63 \%)$ & $0(0 \%)$ & $9(56 \%)$ & $1(6 \%)$ & $1(6 \%)$ & $1(6 \%)$ & $2(13 \%)$ \\
\hline & Primary $(n=26)$ & $26(100 \%)$ & $15(58 \%)$ & $8(31 \%)$ & $13(50 \%)$ & $4(15 \%)$ & $1(4 \%)$ & $2(8 \%)$ & $3(12 \%)$ \\
\hline & Secondary $(\mathrm{n}=108)$ & $89(82 \%)$ & $61(57 \%)$ & $37(34 \%)$ & $24(22 \%)$ & $29(27 \%)$ & $4(4 \%)$ & $6(6 \%)$ & $7(7 \%)$ \\
\hline & Tertiary $(n=45)$ & $26(58 \%)$ & $29(64 \%)$ & $4(9 \%)$ & $7(16 \%)$ & $7(16 \%)$ & $6(13 \%)$ & $0(0 \%)$ & $9(20 \%)$ \\
\hline
\end{tabular}


Perception and Adoption Level of Urban Horticulture Technologies, Nairobi County, Kenya

\begin{tabular}{|c|c|c|c|c|c|c|c|c|c|}
\hline \multirow[b]{3}{*}{ है } & Less than $5,000(n=11)$ & $11(100 \%)$ & $2(18 \%)$ & $0(0 \%)$ & $7(64 \%)$ & $0(0 \%)$ & $0(0 \%)$ & $0(0 \%)$ & $5(46 \%)$ \\
\hline & $5,001-10,000(n=23)$ & $23(100 \%)$ & $12(52 \%)$ & $5(22 \%)$ & $11(48 \%)$ & $0(0 \%)$ & $0(0 \%)$ & $3(13 \%)$ & $3(13 \%)$ \\
\hline & $10,001-15,000(\mathrm{n}=41)$ & $37(90 \%)$ & $26(63 \%)$ & $21(51 \%)$ & $6(15 \%)$ & $0(0 \%)$ & $0(0 \%)$ & $3(7 \%)$ & $4(10 \%)$ \\
\hline$\leq$ & $15,001-20,000(n=39)$ & $24(62 \%)$ & $22(56 \%)$ & $7(18 \%)$ & $10(26 \%)$ & $20(51 \%)$ & $0(0 \%)$ & $2(5 \%)$ & $1(3 \%)$ \\
\hline$\cong$ & $20,001-25,000(n=19)$ & $12(63 \%)$ & $15(79 \%)$ & $6(32 \%)$ & $3(16 \%)$ & $15(79 \%)$ & $0(0 \%)$ & $1(5 \%)$ & $2(11 \%)$ \\
\hline U্ & $25,001-30,000(\mathrm{n}=12)$ & $10(83 \%)$ & $10(42 \%)$ & $4(33 \%)$ & $2(17 \%)$ & $3(25 \%)$ & $4(33 \%)$ & $0(0 \%)$ & $1(8 \%)$ \\
\hline$=$ & $30,001-35,000(\mathrm{n}=16)$ & $14(88 \%)$ & $8(50 \%)$ & $5(31 \%)$ & $2(13 \%)$ & $3(19 \%)$ & $8(50 \%)$ & $0(0 \%)$ & $0(0 \%)$ \\
\hline$\tau$ & $35,001-40,000(n=14)$ & $12(86 \%)$ & $2(14 \%)$ & $0(0 \%)$ & $4(29 \%)$ & $0(0 \%)$ & $0(0 \%)$ & $0(0 \%)$ & $1(7 \%)$ \\
\hline בे & $40,001-45,000(n=6)$ & $6(100 \%)$ & $5(83 \%)$ & $0(0 \%)$ & $4(67 \%)$ & $0(0 \%)$ & $0(0 \%)$ & $0(0 \%)$ & $2(33 \%)$ \\
\hline & $45,001-50,000(n=8)$ & $5(63 \%)$ & $4(50 \%)$ & $1(13 \%)$ & $5(63 \%)$ & $0(0 \%)$ & $0(0 \%)$ & $0(0 \%)$ & $1(13 \%)$ \\
\hline & More than $50,000(n=6)$ & $3(50 \%)$ & $6(100 \%)$ & $0(0 \%)$ & $0(0 \%)$ & $0(0 \%)$ & $0(0 \%)$ & $0(0 \%)$ & $1(17 \%)$ \\
\hline
\end{tabular}

N:B These responses may not add to 100\%, because some respondents gave more than one response(multiple response)

Data from table 5 show that $81 \%$ the respondents grow kales as a major vegetable while $59 \%$ indicated that they grow spinach, $41 \%$ grow onions and $16 \%$ grow green beans. Other crops grown by respondents include tomatoes, carrots, spider plant and cabbage at $11 \%$. On gender, majority of the respondents grew kales at $72 \%$ for female and male at $100 \%$.

\subsection{Method of Technology Transfer of Respondents}

Table6. Distribution frequency on mode of Technology transfer according to social-demographic groups in Kasarani

\begin{tabular}{|c|c|c|c|c|c|c|c|}
\hline & $\begin{array}{c}\text { Socio-economic } \\
\text { characteristics }\end{array}$ & Authority & Tenacity & Experience & Induction & Deduction & $\begin{array}{c}\text { Scientific } \\
\text { Method }\end{array}$ \\
\hline \multirow{3}{*}{ 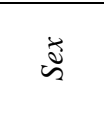 } & Male $(n=34)$ & (11)32\% & (10)29\% & (1)3\% & (3)9\% & (4) $12 \%$ & $(5) 15 \%$ \\
\hline & Female $(n=104)$ & (30)29\% & $(27) 26 \%$ & $(20) 19 \%$ & $(7) 7 \%$ & $(8) 7 \%$ & (12) $12 \%$ \\
\hline & $\operatorname{Total}(\mathrm{n}=138)$ & $(41) 30 \%$ & (37)27\% & $(21) 15 \%$ & $(10) 7 \%$ & $(12) 9 \%$ & (17)12\% \\
\hline \multirow{4}{*}{$\stackrel{\infty}{\infty}$} & Young adults(n=31) & (13) $42 \%$ & $(6) 19 \%$ & $(4) 13 \%$ & $(2) 7 \%$ & $(2) 7 \%$ & $(4) 13 \%$ \\
\hline & Middle aged adults(n=96) & $(25) 26 \%$ & $(28) 29 \%$ & (14) $15 \%$ & $(8) 8 \%$ & (9)9\% & (12)39\% \\
\hline & Older adults $(\mathrm{n}=11)$ & (3) $27 \%$ & (3) $27 \%$ & (3) $27 \%$ & $(0) 0 \%$ & (1) $9 \%$ & (1)9\% \\
\hline & Total $(\mathrm{n}=138)$ & $(41) 30 \%$ & (37)27\% & $(21) 15 \%$ & $(10) 7 \%$ & $(12) 9 \%$ & (17) $12 \%$ \\
\hline \multirow{4}{*}{ 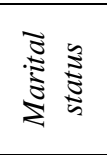 } & Single $(n=13)$ & $(2) 15 \%$ & (3) $23 \%$ & $(5) 39 \%$ & $(1) 7 \%$ & $(1) 7 \%$ & $(1) 7 \%$ \\
\hline & Married(n=98) & (33)34\% & $(25) 26 \%$ & (14) $14 \%$ & $(7) 7 \%$ & $(8) 8 \%$ & (11)11\% \\
\hline & Divorced/separated(n=4) & $(0) 0 \%$ & $(3) 75 \%$ & (1) $25 \%$ & $(0) 0 \%$ & $(0) 0 \%$ & $(0) 0 \%$ \\
\hline & Widowed(n=23) & (6) $26 \%$ & (6) $26 \%$ & (1) $4 \%$ & (2)9\% & (3) $13 \%$ & $(5) 22 \%$ \\
\hline \multirow{4}{*}{ 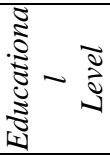 } & Non formal education $(n=8)$ & $(1) 12 \%$ & $(4) 25 \%$ & $(1) 12 \%$ & $(1) 12 \%$ & $(0) 0 \%$ & $(1) 12 \%$ \\
\hline & Primary $(n=16)$ & (3)19\% & $(4) 25 \%$ & (3) $19 \%$ & $(1) 6 \%$ & (3) $19 \%$ & $(2) 13 \%$ \\
\hline & Secondary $(n=41)$ & (13)32\% & (12)29\% & $(6) 15 \%$ & $(3) 7 \%$ & $(2) 5 \%$ & (5) $12 \%$ \\
\hline & Tertiary $(n=73)$ & $(24) 33 \%$ & $(17) 23 \%$ & (11) $15 \%$ & $(5) 7 \%$ & $(7) 10 \%$ & (9) $12 \%$ \\
\hline \multirow{7}{*}{ 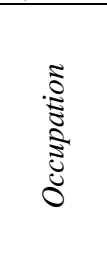 } & Trading $(n=48)$ & (15)31\% & $(15) 31 \%$ & $(4) 8 \%$ & (3) $6 \%$ & (6) $12 \%$ & (5) $10 \%$ \\
\hline & Farming $(n=2)$ & $(0) 0 \%$ & $(1) 50 \%$ & $(0) 0 \%$ & $(0) 0 \%$ & $(0) 0 \%$ & $(1) 50 \%$ \\
\hline & Driving $(\mathrm{n}=3)$ & $(0) 0 \%$ & $(1) 33 \%$ & $(2) 67 \%$ & $(0) 0 \%$ & $(0) 0 \%$ & $(0) 0 \%$ \\
\hline & Civil Service $(n=30)$ & $(8) 27 \%$ & $(10) 33 \%$ & (6)20\% & $(2) 7 \%$ & (3)10\% & (1)3\% \\
\hline & Barbing $(\mathrm{n}=1)$ & $(0) 0 \%$ & $(0) 0 \%$ & $(0) 0 \%$ & (1) $100 \%$ & $(0) 0 \%$ & $(0) 0 \%$ \\
\hline & Unemployed(n=16) & $(4) 25 \%$ & $(4) 25 \%$ & $(2) 13 \%$ & $(2) 13 \%$ & $(1) 6 \%$ & $(3) 19 \%$ \\
\hline & Others $(\mathrm{n}=38)$ & (14)37\% & (6) $16 \%$ & $(7) 18 \%$ & $(2) 5 \%$ & $(2) 5 \%$ & (7) $18 \%$ \\
\hline \multirow{11}{*}{ 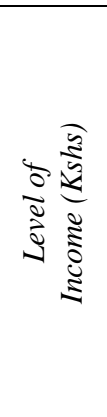 } & Less than $5,000(n=3)$ & $(2) 67 \%$ & $(0) 0 \%$ & $(0) 0 \%$ & $(0) 0 \%$ & (1)33\% & $(0) 0 \%$ \\
\hline & $5,001-10,000(\mathrm{n}=5)$ & (2) $40 \%$ & (1)20\% & $(0) 0 \%$ & (2) $40 \%$ & $(0) 0 \%$ & $(0) 0 \%$ \\
\hline & $10,001-15,000(\mathrm{n}=18)$ & (3) $17 \%$ & (8) $44 \%$ & (3) $16 \%$ & $(1) 6 \%$ & (2) $11 \%$ & (1) $6 \%$ \\
\hline & $15,001-20,000(n=31)$ & (10)32\% & (9)29\% & $(5) 16 \%$ & (1) $3 \%$ & $(5) 16 \%$ & $(1) 3 \%$ \\
\hline & $20,001-25,000(n=28)$ & $(8) 29 \%$ & (5) $18 \%$ & (4)14\% & $(0) 0 \%$ & (4) $14 \%$ & (7) $25 \%$ \\
\hline & $25,001-30,000(n=19)$ & $(7) 37 \%$ & $(5) 26 \%$ & $(4) 21 \%$ & $(2) 11 \%$ & $(0) 0 \%$ & $(1) 5 \%$ \\
\hline & $30,001-35,000(n=13)$ & (3) $23 \%$ & (3) $23 \%$ & $(1) 8 \%$ & $(1) 8 \%$ & $(0) 0 \%$ & $(5) 39 \%$ \\
\hline & $35,001-40,000(\mathrm{n}=9)$ & (3)33\% & (1) $11 \%$ & (3)33\% & $(1) 11 \%$ & $(0) 0 \%$ & (1) $11 \%$ \\
\hline & $40,001-45,000(n=5)$ & $(0) 0 \%$ & (3) $60 \%$ & $(0) 0 \%$ & (1)20\% & $(0) 0 \%$ & $(1) 20 \%$ \\
\hline & $45,001-50,000(n=4)$ & $(2) 50 \%$ & (1) $25 \%$ & $(0) 0 \%$ & (1) $25 \%$ & $(0) 0 \%$ & $(0) 0 \%$ \\
\hline & More than $50,000(n=3)$ & (1)33\% & (1)33\% & (1)33\% & $(0) 0 \%$ & $(0) 0 \%$ & (0)0\% \\
\hline
\end{tabular}

Source: Survey, August 2017

Most of the sampled respondents (41\%) acquired knowledge through authority, Tenacity $27 \%$, and induction $7 \%$. The majority of younger adults (42\%) acquired knowledge through Authority (Table 6 ), while the majority of middle aged adults (29\%) acquired knowledge through Tenacity. On the level of education, the majority $(33 \%)$ of those who had attained tertiary as the highest level of education acquired horticultural technology through authority, while those with non-formal education majority (25\%) acquired through tenacity. 


\subsection{Respondentsby Last Place of Residence}

Table7. Distribution frequency of last place of residence before residing on the current place according to social-demographic groups, Kibera

\begin{tabular}{|c|c|c|c|c|}
\hline & Socio-economic characteristics & Other places in Nairobi & Rural areas & Other urban areas \\
\hline \multirow{3}{*}{ ङँ } & Male $(\mathrm{n}=74)$ & $20(3 \%)$ & $37(50 \%)$ & $17(23 \%)$ \\
\hline & Female $(n=173)$ & $39(23 \%)$ & $84(49 \%)$ & $50(29 \%)$ \\
\hline & Total $(\mathrm{n}=247$ & $59(24 \%)$ & $121(49 \%)$ & $67(27 \%)$ \\
\hline \multirow{3}{*}{$\stackrel{\infty}{\longrightarrow}$} & Young adults $(\mathrm{n}=64)$ & $17(27 \%)$ & $31(48 \%)$ & $16(25 \%)$ \\
\hline & Middle aged $(n=160)$ & $39(24 \%)$ & $75(47 \%)$ & $46(29 \%)$ \\
\hline & Older adults $(\mathrm{n}=23)$ & $3(13 \%)$ & $15(65 \%)$ & $5(22 \%)$ \\
\hline \multirow{4}{*}{ 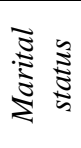 } & Single $(\mathrm{n}=40)$ & $11(28 \%)$ & $22(55 \%)$ & $7(18 \%)$ \\
\hline & Married(n=170) & $36(21 \%)$ & $84(49 \%)$ & $50(29 \%)$ \\
\hline & Divorced/separated $(\mathrm{n}=10)$ & $2(20 \%)$ & $4(40 \%)$ & $4(40 \%)$ \\
\hline & Widowed $(n=27)$ & $10(37 \%)$ & $11(41 \%)$ & $6(22 \%)$ \\
\hline \multirow{4}{*}{ 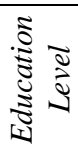 } & Non formal education $(n=23)$ & $7(30 \%)$ & $10(43 \%)$ & $6(26 \%)$ \\
\hline & Primary $(\mathrm{n}=48)$ & $17(35 \%)$ & $21(44 \%)$ & $10(21 \%)$ \\
\hline & Secondary $(n=129)$ & $26(20 \%)$ & $68(53 \%)$ & $35(27 \%)$ \\
\hline & Tertiary $(n=47)$ & $9(19 \%)$ & $22(47 \%)$ & $16(34 \%)$ \\
\hline \multirow{7}{*}{ 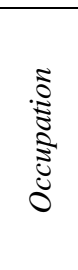 } & $\operatorname{Trading}(\mathrm{n}=101)$ & $25(25 \%)$ & $49(49 \%)$ & $27(27 \%)$ \\
\hline & Farming $(\mathrm{n}=3)$ & $2(67 \%)$ & $0(0 \%)$ & $1(33 \%)$ \\
\hline & Driving $(\mathrm{n}=8)$ & $2(25 \%)$ & $3(38 \%)$ & $3(38 \%)$ \\
\hline & Civil Service $(\mathrm{n}=30)$ & $9(30 \%)$ & $12(40 \%)$ & $8(27 \%)$ \\
\hline & Barbing $(\mathrm{n}=5)$ & $1(20 \%)$ & $3(60 \%)$ & $1(20 \%)$ \\
\hline & Unemployed $(n=36)$ & $6(17 \%)$ & $22(61 \%)$ & $9(25 \%)$ \\
\hline & Others $(\mathrm{n}=64)$ & $14(22 \%)$ & $6(9 \%)$ & $5(8 \%)$ \\
\hline \multirow{11}{*}{ 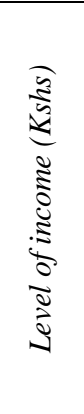 } & Less than $5,000(n=20)$ & $4(20 \%)$ & $14(70 \%)$ & $2(10 \%)$ \\
\hline & $5,001-10,000(n=16)$ & $5(31 \%)$ & $5(31 \%)$ & $6(38 \%)$ \\
\hline & $10,001-15,000(n=48)$ & $11(23 \%)$ & $26(54 \%)$ & $11(23 \%)$ \\
\hline & $15,001-20,000(n=42)$ & $11(26 \%)$ & $21(50 \%)$ & $10(24 \%)$ \\
\hline & $20,001-25,000(n=38)$ & $8(21 \%)$ & $18(47 \%)$ & $12(32 \%)$ \\
\hline & $25,001-30,000(n=19)$ & $3(16 \%)$ & $9(47 \%)$ & $7(37 \%)$ \\
\hline & $30,001-35,000(n=14)$ & $6(43 \%)$ & $6(43 \%)$ & $2(14 \%)$ \\
\hline & $35,001-40,000(n=17)$ & $4(24 \%)$ & $8(47 \%)$ & $5(29 \%)$ \\
\hline & $40,001-45,000(\mathrm{n}=9)$ & $2(22 \%)$ & $4(44 \%)$ & $3(33 \%)$ \\
\hline & $45,001-50,000(n=13)$ & $2(15 \%)$ & $7(54 \%)$ & $4(31 \%)$ \\
\hline & More than $50,000(n=11)$ & $1(9 \%)$ & $7(64 \%)$ & $3(27 \%)$ \\
\hline
\end{tabular}

Source: Survey, August 2017

As shown in Table 7 above, $49 \%$ came from the rural areas, $27 \%$ from other locations of urban areas, and $24 \%$ from other places within Nairobi. The majority of younger adults and middle aged adults came from rural areas at $48 \%$ and $47 \%$ respectively, while $13 \%$ of older adults came from other places in Nairobi areas.

\section{SUMMARY AND CONCLUSIONS}

\subsection{Respondents Characteristics}

It is apparent that the majority of respondents were females. This suggests that urban horticulture is dominated by females who in most cases are married with household care giving responsibilities. This agrees with (Lee-Smith and Prain., 2010) who indicated that in sub-Saharan Africa, studies of urban agriculture have been limited, but those that have been done generally suggest that approximately one-third of households are engaged in some form of urban agriculture, and that two third of the farmers are women. Urban farming also favors both social inclusion and reduction of gender inequalities in cities as 65\% of urban farmers are women (Orsini et al. 2013). It is because of the close proximity to the home, gardening can be much better combined with child care which is still seen as a woman's duty in many countries (Dubbeling, de Zeeuw and van Veenhuizen, 2010). (Barau and Oladeji, 2017) found that $69.4 \%$ of the females who were doing farming were married in Sokoto Metropolis, Nigeria.

It is clear that the majority of the respondents are middle aged adults and education level ranging from informal to post-secondary. Accessing land for farming in urban areas requires energy, determination and maturity. The finding agrees with (Barau and Oladeji, 2017) who found that most of the urban women farmers (38.9\%) were in the active age range and also (Teig et al., 2009) found that the 
majority of community gardeners are seniors. Age is also assumed to be a determinant of adoption of new technology. Older farmers are assumed to have gained knowledge and experience over time and are better able to evaluate technology information than younger farmers (Kariyasa \& Dewi, 2013). On the other hand, age has been found to have a negative relationship with adoption of technology. This relationship is explained by Thomas et al., (2017) that as farmers grow older, there is an increase in risk aversion and a decreased interest in long term investment in the farm. While the, younger farmers are typically less risk-averse and are more willing to try new technologies. On education, it is evident from the findings that the farmers are spread all over education level. The findings outlined are in agreement with the observation in Accra, urban farmers interviewed had no particular educational pattern (World Bank, 2013).

\subsection{Space Identified for Production of Vegetables}

A renovated urban farming arose worldwide as a response to a number of factors (Bohn and Viljoen 2011). In city centres, the inadequate space has been a major challenge in the diffusion of agricultural activities (Christine \& Nazim G. 2015). In order to utilize the available vacant urban spaces as efficiently as possible, new cultivation methods are required (Christine \& Nazim G. 2015). As a result, the introduction of horticulture activities in available spaces in cities has recently been observed in both land-based and non-land-based vacant spaces. First, non-constructed areas (e.g., abandoned plots, green spaces or interstitial areas) are being converted into urban gardens when available and vacant. Second, innovative methods for turning concrete into urban green infrastructures for vegetable production have been developed in the recent past, ranging from vertical farms (Despommier, 2011) to the most ordinary rooftop gardens. The study carried in Nairobi found that urban agriculture is practiced in backyard farms, on open spaces under power lines, along roadsides, along railway lines and riverbanks as well as on institutional land (World bank, 2013).Vacant spaces in cities should also be considered as possible alternative of reducing pressure from rural agriculture and to decompensate land loss (Christine \& Nazim G. 2015), by turning vacant lots into urban vegetable gardens, food security and sustainability are increased.

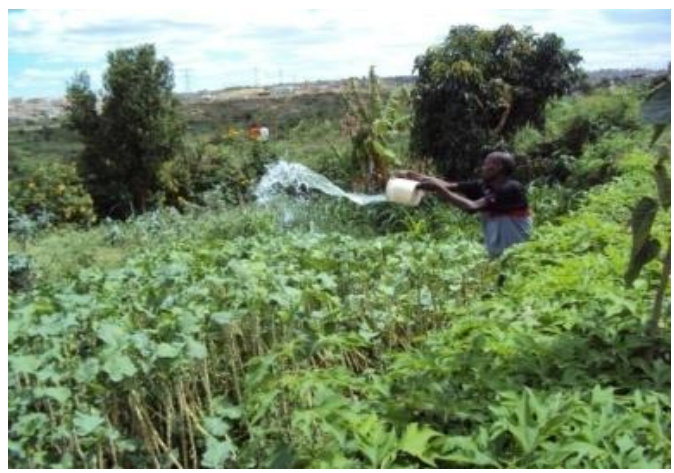

Fig1. A farmer watering sukuma wiki at Mwiki Source: Survey, August 2017

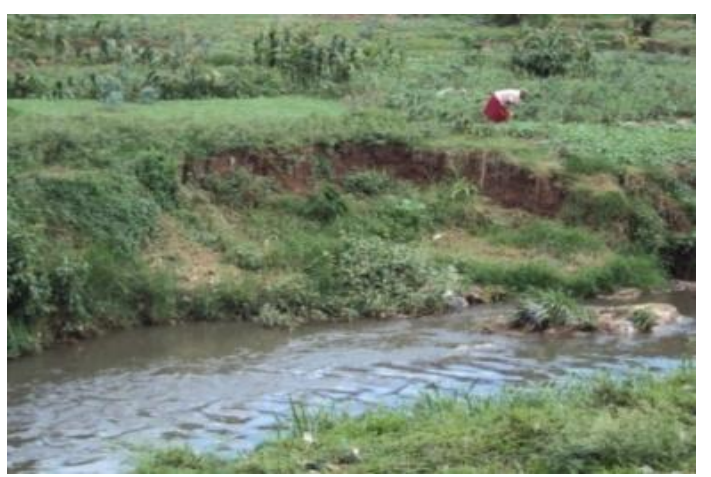

Fig2. A farmer attending to cowpeas at Mwiki Source: Survey, August 2017.

Gender, age, marital status, education level, occupation and level of income had non-significant to space where production of vegetables is done. This may suggest that due to limited space in urban areas for farming, farmers have no choice other than using the available space.

\subsection{Reason for Adoption of Urban Technologies}

Golden (2013) found that urban dwellers can benefit from urban farming through accessing land, community development, cross-generational and cultural integration, job creation, and economic savings on food. Municipal authorities can also benefit from urban agriculture through savings (Chaminuka \& Dube 2017). Land for farming is limited in urban areas, urban farming creates access to land by creating space within cities for farming. Urban agriculture can benefit urban dwellers through accessing land for them to call their own, thereby creating some sense of pride through ownership of the land (Chaminuka \& Dube 2017).

Food access and availability are important dimensions that constitute food security (Chaminuka \& Dube 2017). Urban horticultural has been viewed as an intervention to deal with food security. It has been used as an effective means for improving food security in critical and insecure areas (Corrigan, 2011; Larsen \& Gilliland, 2009). Matteson (2007) found that, above 700 community gardens exist in 
New York City, which have increased food access and availability to urban dwellers. Apart from improving food access and availability, urban agriculture is also important in job creation. Hagey, Rice, S. \& Flournoy (2012) indicates that urban agriculture that offers packaging and processing, to complement crop cultivation is capable of creating many jobs for urban residents. Metcalf and Widener (2011) argues that through Job Creation, many urban agriculture projects will engage youths to manage horticultural farms and this will provides them with income above skills training. Urban agriculture can provide savings for county government. For example, the management of vacant lots by communities in San Francisco turned into urban farming areas benefited the Department of Public Works about US\$4,100 through preventing vandalism, dumping, and labor-intensive upkeep (SPUR, 2012).

Out of 6 variables studied, occupation in Kasarani, age and level of income in Mathare were significant $(\mathrm{P}<0.05)$ on adoption of horticultural technologies. Non-significant of gender, marital status and education level observed in this study may indicate that these socio-economic variables definitely do not have any bearing on the adoption of technologies. Majority of the respondents were traders, which may indicate they have flexible time to attend to their crops.

\subsection{Challenges Farmers Face in Adoption of Urban Farming Technologies}

Limited access to land, lack of tenure on property, and insufficient infrastructure and services for urban growers are among the main restrictions of urban horticulture according to (Lovell, 2010). Land tenure affects the application of technologies for agricultural (Islam \& Tuulikki, 2009), secured land tenure gives sufficient incentives to the farmers to increase their efficiencies in terms of production. Without secured property rights farmers do not feel emotional attachment to the land they cultivate, do not invest in land development and will not use inputs efficiently (Islam \& Tuulikki, 2009). Theft of crops by non-farmers and stray animals eating crops, are other problems associated with to urban farming (Chaminuka \& Dube, 2017).

Urban agriculture is occasionally practiced in public areas unsuitable for housing, such as road verges, banks of drainage channels, wetlands and contaminated sites such as scrap yards and dumpsites for solid and liquid wastes (Nabulo et al., 2008). As such the farmers' crop has at times been slashed down by authorities (Chaminuka \& Dube, 2017).

\subsection{Kind of Vegetables Grown}

Majority of the respondents mentioned kales and spinach. The producers in urban areasgrow more green leafy vegetables, such as kale and spinach (World Bank, 2013) this also agrees with (Gallaher et al., 2015, who indicated that over the past several years, in urban areas especially, indigenous vegetables have been replaced by kale, swisschard, and cabbage. Kales (Sukuma wiki) literally means "to push the week". As the name suggests, low income earners can survive on it by making it a daily meal. Kales due to its low price, it keeps people going hoping for better tomorrow.

The case study in Kibera, households and other institutions like the eco school cultivate crops such as kale and spinach - leafy vegetables which adapts very well to the conditions of sack farming (Erulkar \& Matheka 2007).

\subsection{Last Place of Residence}

Most respondents came from rural areas as a result most households engaging in urban agriculture could be bringing to the city the rural culture of farming to urban areas. Through urban farming, migrants gets opportunity to grow food for consumption purposes and may even sell surpluses (Beckie \& Bogdan, 2010), Migrants have important skills and culture which they can share with urban farmers.(Gallaher et al., 2015) found out that the majority of farmers and non-farmers (85\% and 75\%, respectively) have had previous experience with mixed farming in rural areas, mostly before they migrated to Kibera. A report by World Bank (2013) indicates that most of the residents engaged in urban farming had stayed in the city the longest or always lived there.

\section{REFERENCES}

[1] Alaimo K, Packnett E, Milers RA, Kruger DJ. 2008. Fruit and vegetable intake among urban community gardeners. J Nutr Educ Behav 40(2):94-101.

[2] Barau A. A. and Oladeji D. O. (2017) "Participation of Urban Women in Agricultural Production Activities in the Sokoto Metropolis, Nigeria," Journal of Natural Resources and Development, pp. 8490, 2017.Doi: https://doi.org/10.5027/jnrd.v7i0.10 
[3] Baumgart-Getz A. L.S. Prokopy, and K. Floress (2012). Why farmers adopt best management practices in the United States: a meta-analysis of the adoption literature. J. Environ. Manag., 96 (1), pp. 1725, 10.1016/j.jenvman.2011.10.006

[4] Beckie, M. and Bogdan, E. (2010). Planting roots: Urban agriculture for senior immigrants. Journal of Agriculture, Food Systems, and Community Development, 1(2): 77-89. https://doi:10.5304/jafscd. 2010. 012.004

[5] Bertrand, M and Bouchard, S (2008) applying the technology acceptance model to VR with people who are favourable to its use. Journal of Cyber Therapy \& Rehabilitation. Volume 1(1), 200-210.

[6] Birhanu M. (2018);A Review on Factors Affecting Adoption of Agricultural New Technologies in Ethiopia; J AgriSci Food Res, Vol 9(3):

[7] Bohn K, and Viljoen A (2011) The edible city: envisioning the continuous productive urban landscape. Field J 4(1):149-161

[8] Chaminuka N. \& Dube E. (2017). Urban agriculture as a food security strategy for urban dwellers: A case study of Mkoba residents in the city of Gweru, Zimbabwe. International Journal of Social Sciences. Regular Issue Volume 3 Issue 2, pp. 26 - 45. doi-https://dx.doi.org/10.20319/pijss.2017.32.2645

[9] Christine E. \& Nazim G. (2015); Urban vegetable for food security in cities. A review; Agron. Sustain. Dev. (2015) 35:483-498: DOI 10.1007/s13593-014-0273-y

[10] Cohen, M. J., \& Garrett, J. L. (2010).The food price crisis and urban food (in)security. Environment and Urbanization, 22(2), 467-482.

[11] Corrigan, M.P. (2011). Growing what you eat: Developing community gardens in Baltimore, Maryland. Applied Geography, Vol. 31(4), 1232-1241.https://doi.org/10.1016/j.apgeog.2011.01.017

[12] Desgroppes, A., \& Taupin, S. (2011). Kibera: Thebiggest slum in Africa? Les Cahiers de l'Afriquedel'Est, 44, 23-34. Retrieved from Sciences del'Hommeet de la Société (HAL) website: https://halshs.archivesouvertes.fr/halshs-00751833

[13] Despommier, D. (2011). The vertical farm: Controlled environment agriculture carried out in tall buildings would create greater food safety and security for large urban populations. J. frVerbrauch., 6, 233-236.Doi: https://www.researchgate.net/publication/226426887

[14] Dubbeling M, de Zeeuw H, van Veenhuizen R (2010). Cities, poverty and food-multi-stakeholder policy and planning in urban agriculture. RUAF Foundation, Rugby, p 173

[15] Ducey\& Adam J.(2013)"Predicting Tablet Computer Use: An Extended Technology Acceptance Model" Graduate Theses and Dissertations. Retrieved on 01/03/2015, from http://scholarcommons.usf.edu/etd/4471

[16] Erulkar A.S. and Matheka J.K. (2007). Adolescence in the Kibera Slums of Nairobi, Kenya, Population Council, Nairobi

[17] FAO (Food and Agricultural Organization), (2012) Growing Greener Cities in Africa, FAO: Rome

[18] FAO, (2010). The State of Food Insecurity in the World.

[19] Flora, C.B., Bregendahl, C. and Fey, S. (2007) Mobilizing internal and external resources for rural community development, in: R.D. Knutson, S.D. Knutson and D.P. Ernstes (eds) Perspectives on 21st Century Agriculture: A Tribute to Walter J. Armbruster. Chicago, IL: The Farm Foundation, pp. 210-220

[20] Gallaher, C. M., WinklerPrins, A. M. G. A., Njenga, M., \& Karanja, N. K. (2015). Creating space:Sack gardening as a livelihood strategy in the Kibera slums of Nairobi, Kenya. Journal of Agriculture, Food Systems, and Community Development. Advance online publication.http://dx.doi.org/10.5304/jafscd.2015. 052.006

[21] Galhena, D. H., Freed, R., \&Maredia, K. M. (2013). Home gardens: a promising approach to enhance household food security and wellbeing. Agriculture \& Food Security, 2(1), 8.

[22] Genter C, Roberts A, Richardson J, Sheaff M. (2015)The contribution of allotment gardening to health and wellbeing: A systematic review of the literature. British Journal of Occupational Therapy. 2015; 78 (10) : 593-605.

[23] Golden, S. (2013). Urban agriculture impacts: Social, health, and economic: A Literature review. University of California: California

[24] Gillespie, G., D.L. Hilchey, C.C. Hinrichs, and G. Feenstra (2007).Farsmers' Markets as Keystones in Rebuilding Local and Regional Food Systems. Ln C.C. Hinrichs and T.A. Lyson, eds. Remaking the North American Food System: Strategies for Sustainability. Lincoln, NE: University of Nebraska Press, pp. 65-83.

[25] Hagey, A., Rice, S. \& Flournoy, R. (2012). Growing urban agriculture: Equitable strategies and policies for improving access to healthy food and revitalizing communities. Policy Link. Retrieved 06 November 2016, from www.policylink.org

[26] International Food Policy Research Institute. 2014. Washington, DC: Global Nutrition Report 2014 : actions and accountability to accelerate the world's progress on nutrition. 
[27] Islam K.M. \& Tuulikki P. (2009) .Effects of land tenure and property rights on agricultural productivity inEthiopia, Namibia and Bangladesh.Department of Economics and Management, University of Helsinki,2009 J. Environ. Manag, 96 (1) (2012), pp. 17-25, 10.1016/j.jenvman.2011.10.006

[28] Jain, R., Arora, A., \& Raju, S. (2009). A novel adoption index of selected agricultural technologies: Linkages with infrastructure and productivity. Agricultural Economics Research Review, 22(1), 109-120.

[29] Kariyasa, K. and Dewi, Y.A. (2013) Analysis of Factors Affecting Adoption of Integrated Crop Management Farmer Field School Estimation: (Icm-Ffs) in Swampy Areas. International Journal of Food and Agricultural Economics, 1, 29-38.

[30] Kenya National Bureau of Statistics (KNBS). (2010). 2009 Kenya Population and Housing Census. Vol.1A. Ministry of Planning and National Development, Nairobi, Kenya

[31] Larsen, K., \& Gilliland, J. (2009). A farmers market in a food desert:Evaluating impacts on the price and availability of healthy food. Health \& Place, Vol. 15(4):1158-1162.doi:10.1016/j.healthplace.2009.06.007

[32] Lee-Smith, D. and Prain, G. (2010).The contribution of research-development partnerships to building urban agriculture policy. In: Prain, G., Karanja, N., Lee-Smith, D. (Eds.), Urban Harvest: Agriculture in the cities of Cameroon, Kenya and Uganda. International Potato Center (CIP), Rome, Italy. pp 287-308

[33] Loevinsohn, M., Jim Sumberg, J., Diagne, A. and Whitfield, S. (2013). Under what circumstances and conditions does adoption of technology result in increased agricultural productivity? A Systematic Review Prepared for the Department of International Development. Institute of Development Studies, Brighton, UK.[http://r4d.dfid.gov.uk/pdf/outputs/systematicreviews/Productivity_systematic_review_report_Loevins ohn.pdf] site visited on 02/02/2014.

[34] Lovell ST (2010) Multifunctional urban agriculture for Sustainable land use planning in the United States. Sustainability 2:2499-2522. https://doi: 10.3390/su2082499

[35] Makokha, G. L., and C. A. Shisanya (2010). Trends in Mean Annual Minimum and Maximum Near Surface Temperature in Nairobi City, Kenya. Advances in Meteorology, 2010: 6.

[36] Matteson, K.C. (2007). Diversity and conservation of insects in urban gardens: theoretical and applied implications. Dissertation, Fordham University, Bronx, New York.208 pp.

[37] Metcalf, S.S., \& Widener, M.J. (2011). Growing buffalo's capacity for local food: A systems framework for sustainable agriculture. Applied Geography, Vol. 31(4): 1242-1251.Doi: 10.1016/j.apgeog.2011.01. 008

[38] Mohammadi Jamal, and Soleimani Shiri Mortez (2014). The Effect of Urban Agriculture in Urban Sustainable Development and Its Techniques: A Case Study in Iran, International Journal of Agriculture and Forestry, Vol. 4 No. 4, pp. 275-285. doi: 10.5923/j.ijaf.20140404.03.

[39] Nabulo, G, Nasinyama, G, Lee-Smith, D \& Cole, D 2004, 'Gender analysis of urban agriculture in Kampala, Uganda' in Urban Agriculture Magazine No. 12 - Gender and Urban Agriculture, Resource Centre on Urban Agriculture and Forestry (RUAF), http://www.ruaf.org/no12/32_33.pdf [Accessed 19 February 2008].

[40] de Neergard A, Drescher AW, Kouamé C (2009) Urban and peri-urban agriculture in African cities. In: Pasquini CM, Drescher MW, Shackleton AW (eds) African indigenous vegetables in urban agriculture. Earthscan, London, pp 35-58

[41] Nikolaidou, S., Klöti, T., Tappert, S., \& Drilling, M. (2016). Urban Gardening and Green Space Governance: Towards New Collaborative Planning Practices. Urban Planning, 1(1), 5-19. https://doi.org/ 10.17645/up.v1i1.520

[42] Orsini F, Kahane R, Nono-Womdim R, Gianquinto G (2013a) Urban agriculture in the developing world: a review. Agron Sustain Dev 33:695-720. https://doi: 10.1007/s13593-013-0143

[43] Orsini, F.; Gasperi, D.; Marchetti, L.; Piovene, C.; Draghetti, S.; Ramazzotti, S.; Bazzocchi, G.; Gianquinto, G (2013b) Exploring the production capacity of rooftop gardens (RTGs) in urban agriculture: The potential impact on food and nutrition security, biodiversity and other ecosystem services in the city of Bologna. Food Secur. 2014, 6, 781-792.

[44] Park, Y., Quinn, J., Florez, K., Jacobson, J., Neckerman, K., \& Rundle, A. (2011). Hispanic immigrant women's perspective on healthy foods and the New York City retail food environment: A mixed-method study. Social Science \& Medicine, Vol. 73(1): 13-21. Doi: 10.1016/j. socscimed.2011.04.012

[45] Rezai, G.; Shamsudin, M.N.; Mohamed, Z. (2016) Urban agriculture: A way forward to food and nutrition security in Malaysia. Procedia-Soc. Behav. Sci. 216, 39-45.

[46] Ruth Stewart, Marcel Korth, Laurenz Langer, Shannon Rafferty, Natalie Rebelo Da Silva and Carina van Rooyen (2013). Environmental Evidence. The official journal of the Collaboration for Environmental Evidence20132: https://doi.org/10.1186/2047-2382-2-7 
[47] Shyr, C.L. \& Reily, H.E.( 2017). Introductory Horticulture, 9th ed. Gengage Learning, Stamford, USA, P. 5. ISBN 978-12854-2472-9

[48] SPUR (2012). Public harvest. SPUR Report, 1-36.

[49] Teo, T. (2013).A comparison of non-nested models in explaining teachers' intention to use technology. British Journal of Educational Technology, Vol.44 (3), E81-E84. doi:10.1111/j.1467-8535. 2012.01350.x

[50] Teig, E., Amulya, J., Bardwell, L., Buchenau, M., Marshall, J. A., \&Litt, J. S. (2009). Collective efficacy in Denver, Colorado: Strengthening neighborhoods and health through community gardens. Health \& Place, 15(4), 1115-1122.

[51] Thomas B, Zhuang J, Owusu S, Mensah and Ayamba E (2017). Factors Influencing the Agricultural Technology Adoption: The Case of Improved Rice Varieties (Nerica) in the Northern Region, Ghana. Journal of Economics and Sustainable Development. Vol.8, No.8, 2017

[52] Uaiene, R., Arndt, C., Masters, W. (2009) Determinants of Agricultural Technology Adoption in Mozambique. Discussion papers No. 67E

[53] UNICEF ( 2014) Integrated Health and Nutrition Baseline Survey in the Nairobi slums

[54] United Nations, Department of Economic and Social Affairs, Population Division (2015). World Population Prospects: The 2015 Revision. New York: United Nations.

[55] United Nations, Department of Economic and Social Affairs, Population Division (2014). World Population Prospects: The 2014 Revision. New York: United Nations.

[56] Wakefield S, Yeudall F, Taron C, Reynolds J, Skinner A (2007).Growing urban health: community gardening in South-East Toronto. Health Promot Int. 2007 Jun; 22(2):92-101.

[57] World Bank (2013). Urban Agriculture: Findings from Four City Case Studies. Urban Development Series Knowledge Papers, Volume 18 (Dec 2010). Washington, USA. http://www.worldbank.org/en/topic/ urbandevelopment/publication/urban-agriculture-four-city-case-studies

[58] World Bank Group. (2008).Agriculture for Development. (C) World Bankhttps://siteresources. worldbank. org/INTWDR2008/Resources/WDR_00_book.pdf

[59] World Bank Group. 2018. Kenya Economic Update, April 2018, No. 17 : Policy Options to Advance the Big 4. World Bank, Nairobi. (C) World Bank. https://openknowledge.worldbank.org/handle/10986/29676 License: CC BY 3.0 IGO."

Citation: Ezra Oyaro, J. B Mukundi, "Perception and Adoption Level of Urban Horticulture Technologies, Nairobi County, Kenya", International Journal of Forestry and Horticulture (IJFH), vol. 5, no. 2, pp.19-35, 2019. Available: DOI: http://dx.doi.org/10.20431/2454-9487.0502003

Copyright: (C) 2019 Authors. This is an open-access article distributed under the terms of the Creative Commons Attribution License, which permits unrestricted use, distribution, and reproduction in any medium, provided the original author and source are credited. 